ARTICLE

https://doi.org/10.1038/s41467-019-14058-5

\title{
Single cell and genetic analyses reveal conserved populations and signaling mechanisms of gastrointestinal stromal niches
}

Ji-Eun Kim (10) 1,2, Lijiang Fei ${ }^{3}$, Wen-Chi Yin 1,2, Sabrina Coquenlorge ${ }^{1,2}$, Abilasha Rao-Bhatia 1,2, Xiaoyun Zhang ${ }^{1,2}$, Sammy Shun Wai Shi ${ }^{4,5}$, Ju Hee Lee ${ }^{4,5}$, Noah A. Hahn1,2, Wasi Rizvi ${ }^{1,2}$, Kyoung-Han Kim (D) 6,7, Hoon-Ki Sung 4,5, Chi-chung Hui, ${ }^{1,2}$ Guoji Guo ${ }^{3}$ \& Tae-Hee Kim (i) ${ }^{1,2 \star}$

Stomach and intestinal stem cells are located in discrete niches called the isthmus and crypt, respectively. Recent studies have demonstrated a surprisingly conserved role for Wnt signaling in gastrointestinal development. Although intestinal stromal cells secrete Wnt ligands to promote stem cell renewal, the source of stomach Wnt ligands is still unclear. Here, by performing single cell analysis, we identify gastrointestinal stromal cell populations with transcriptome signatures that are conserved between the stomach and intestine. In close proximity to epithelial cells, these perictye-like cells highly express telocyte and pericyte markers as well as Wnt ligands, and they are enriched for Hh signaling. By analyzing mice activated for Hh signaling, we show a conserved mechanism of GLI2 activation of Wnt ligands. Moreover, genetic inhibition of Wnt secretion in perictye-like stromal cells or stromal cells more broadly demonstrates their essential roles in gastrointestinal regeneration and development, respectively, highlighting a redundancy in gastrointestinal stem cell niches.

\footnotetext{
${ }^{1}$ Program in Developmental \& Stem Cell Biology, The Hospital for Sick Children, Toronto, ON M5G 0A4, Canada. ${ }^{2}$ Department of Molecular Genetics, University of Toronto, Toronto, ON M5S 1A8, Canada. ${ }^{3}$ Center for Stem Cell and Regenerative Medicine, Zhejiang University of School of Medicine, Hangzhou 310058, China. ${ }^{4}$ Translation Medicine Program, The Hospital for Sick Children, Toronto, ON M5G OA4, Canada. ${ }^{5}$ Department of Laboratory Medicine and Pathobiology, University of Toronto, ON M5S 1A8, Canada. ${ }^{6}$ University of Ottawa Heart Institute, Ottawa, ON K1Y 4W7, Canada.

${ }^{7}$ Department of Cellular and Molecular Medicine, University of Ottawa, Ottawa, ON K1H 8M5, Canada. *email: tae-hee.kim@sickkids.ca
} 
M esenchymal-epithelial cross talk plays a crucial role in organ specification and development ${ }^{1,2}$. Tissue grafting studies showed that tissue-specific mesenchymal signals influence gut endoderm differentiation ${ }^{3}$. Indeed, differential gene expression analysis of the mouse embryonic stomach and intestine identified a stomach mesenchyme-specific factor, Barxl, and its deletion caused a striking intestinal transformation, highlighting the significance of organ-specific stromal niche signals ${ }^{4}$. Consistent with these distinct organ niche components, adult stomach, and intestinal epithelial cells are maintained by the activity of stem cells located in discrete epithelial niches, the isthmus and crypt, respectively ${ }^{5,6}$. Wnt signaling plays a critical role in tumorigenesis, adult stem cell homeostasis, and hind gut specification during development ${ }^{7}$. Although thought to be restricted to the intestine and suppressed in the stomach ${ }^{4,8}$, Wnt signaling has recently been shown to perform a surprisingly conserved function in stomach stem cells and their development 9 However, how and where Wnt ligands in the stomach are produced is unknown ${ }^{10}$.

In contrast to stomach stem cell niches, intestinal stem cell niches have been extensively investigated. Intestinal organoids depend entirely upon Paneth cells, which secrete stem cell niche signals that include Wnt $3 \mathrm{a}^{11}$. Despite this fact, Wnt $3 a$ deletion and ablation of Paneth cells in vivo yielded, surprisingly, no obvious stem cell defects, suggesting another sources of stem cell niche signals ${ }^{12-14}$. Genetic inhibition of Wnt secretion from epithelial cells consistently failed to induce stem cell defects ${ }^{15,16}$, while its global inhibition led to the loss of stem cell proliferation $^{17}$. Indeed, additional mouse genetic studies demonstrated that FoxL1+, Pdgfr- $\alpha+$, and CD34+ mesenchymal cells constitute a critical intestinal stem cell niche that secretes Wnt ligands ${ }^{18-20}$. Most recently, it has been shown that FoxL1 expressing cells represent telocytes, which are in proximity to intestinal epithelial cells, while PDGF- $\alpha$ is more broadly expressed throughout the gut mesenchyme ${ }^{21}$. Interestingly, genetic inhibition of Wnt secretion from aSMA+ myofibroblasts led to no obvious stem cell defects ${ }^{15}$. Despite these significant and complex roles of intestinal stromal cells in stem cell homeostasis, their heterogeneity has not been fully investigated. Of note, Hedgehog (Hh) signaling is known to be active in pericryptal cells, and genetic inhibition of Wnt secretion from these $\mathrm{Hh}$ responsive cells arrested colonic stem cells, suggesting its role in the regulation of intestinal stem cell niche signals ${ }^{17,22}$. Interestingly, Helicobacter pylori promotes stomach epithelial proliferation by increasing the expression of $R$-spondin 3, a Wnt agonist ${ }^{23}$. Given the surprisingly conserved role of Wnt signaling in both the stomach and intestine, we hypothesized that stomach stromal cells also play a critical role as a stem cell niche.

To determine the role of stomach stromal stem cell niches and define the heterogeneity of both stomach and intestinal stromal cells, we performed single cell transcriptome analysis and identified conserved stromal cell populations that are Hh responsive and that express Wnt ligands and telocyte markers. Our genetic analysis demonstrated the critical role of these cell populations in gastrointestinal regeneration, while revealing their redundancy with other stromal cell populations, such as CD31-positive endothelial and Ly6c-positive stromal cells during development and adult homeostasis. Moreover, our transcriptional analysis shows Hh- and GLI2-mediated transcriptional activation of stem cell niche signals that is conserved in both the stomach and intestine.

\section{Results}

Conserved gastrointestinal stromal populations. To define the heterogeneity of both stomach and intestinal stromal stem cell niches, we isolated gastrointestinal stromal cells from mice with Bap $x 1^{+/ C r e}$ and Rosa26 $6^{\text {tdTomato }}$ alleles, which broadly label gastrointestinal stromal cells, though not those of the enteric nervous system ${ }^{24}$. We then analyzed their transcriptomes at the single cell level (4946 for stomach and 3459 for intestine each) by performing Drop-Seq (Supplementary Fig. 1) ${ }^{25}$. T-distributed stochastic neighbor embedding ( $\mathrm{t}-\mathrm{SNE}$ ) analysis identified more than 10 different stromal cell clusters each for the stomach and intestine (Fig. 1a-d; Supplementary Figs. 1-4, Supplementary Data 1). Furthermore, our unsupervised hierarchical analysis identified stromal cell clusters expressing either conserved or distinct transcriptome signatures between the stomach and intestine (Fig. 1e; Supplementary Figs. 5 and 6). The populations of stromal fibroblast cells (St C1, 2, 3, 6, 9 and Int C1-6), pericytelike stromal cells (telocyte; St C5, 7 and Int C8), lymphatic endothelial cells (St C11 and Int C7) and pericytes (St C10 and Int C11) expressed similar gene signatures; conversely, mesothelial cells (St C15 and Int C12) and macrophage-like cells (St C14 and Int C10) showed distinct gene expression patterns (Fig. 1a-e; Supplementary Data 1).

To define the stromal cells expressing stem cell niche signals, we analyzed Wnt ligand expression by performing Markov affinitybased graph imputation of cells (MAGIC) ${ }^{26}$. Many different stromal cell populations expressed Wnt ligand and agonist (i.e., Rspo) genes, indicating potentially redundant sources of Wnt niche signals (Supplementary Figs. 7-9). We also found the expression of Wnt antagonist (i.e., $D k k$ ) genes in gut stromal cell populations (Supplementary Fig. 10). Of note, the stromal cell populations conserved between the stomach and intestine were more highly enriched for the Wnt pathway, compared to the distinct cell populations (Fig. 1f, g), while some unconserved stromal populations also expressed Wnt ligands (Supplementary Fig. 11). In addition, these conserved cell populations showed robust expression of pericyte markers such as Ng2 (Cspg4) and $P d g f r-\beta$ (Fig. 2a, b; Supplementary Fig. 12) ${ }^{27}$. Intestinal pericryptal cells located near the stem cells have been implicated in stromal stem cell niche function ${ }^{17-20}$. Our single cell analysis showed that known markers, such as CD34, $P d p n$, and $P d g f r-\alpha$, of these intestinal pericryptal cells are broadly expressed in gastrointestinal stromal cells, which include the conserved populations (Supplementary Figs. 12 and 13). To validate $\mathrm{Ng} 2$ expression in vivo, we utilized Ng2 $2^{+/ D s R e d}$ and Ng2-Cre;Rosa26 $6^{+/ t d T o m a t o}$ mice, and performed co-labeling immunofluorescence stainings. We found that pericyte markers (Desmin, PDGFR $\beta$, and Nestin) and a villus core stromal cell marker, PDGFRa, but not glial cell markers (s100 $\beta$ and GFAP) are expressed in Ng2+ conserved stromal cell populations (Supplementary Figs. 14 and 15). While these cells still express some pericyte markers, they failed to colocalize with CD31+ endothelial cells (Supplementary Fig. 15) and expressed genes distinct from those of gastrointestinal pericytes (Supplementary Fig. 16). Therefore, we will refer to them as pericyte-like stromal cells.

Interestingly, a recent paper has shown that FoxL1 is expressed in telocytes, which constitute a key intestinal stem cell niche ${ }^{21}$, but their role in the stomach as a stem cell niche has been unexplored. Our analysis demonstrated a highly overlapping expression pattern of Foxl1 and Ng2 (Cspg4) in cell clusters conserved between the stomach and intestine (Fig. 2a-c). Corroborating this data, our analysis of the available data set from a recently published Foxl1+ telocyte paper confirmed exclusive expression of $\mathrm{Ng} 2$ in Foxl1 + cells (Fig. 2d). Indeed, our analysis of $\mathrm{Ng} 2^{+/ D s R e d}$ mice showed that $\mathrm{Ng} 2$ is highly expressed in pericryptal cells in proximity to gastrointestinal epithelial cells (Fig. 2e). Moreover, single-molecule RNA fluorescence in situ hybridization (smFISH) analysis confirmed the overlapping expression of $\mathrm{Ng} 2$ and Foxl1 in vivo, indicating that our identified conserved pericyte-like cell 
a
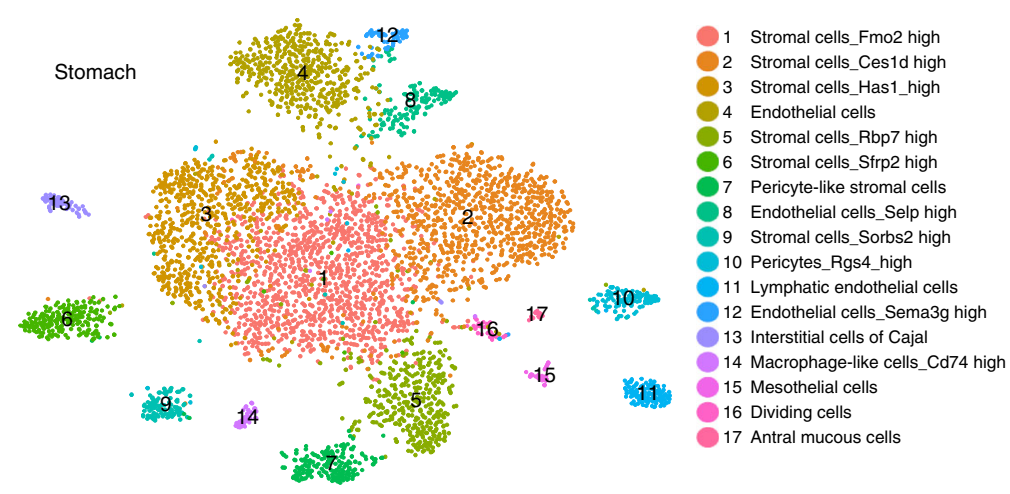

C
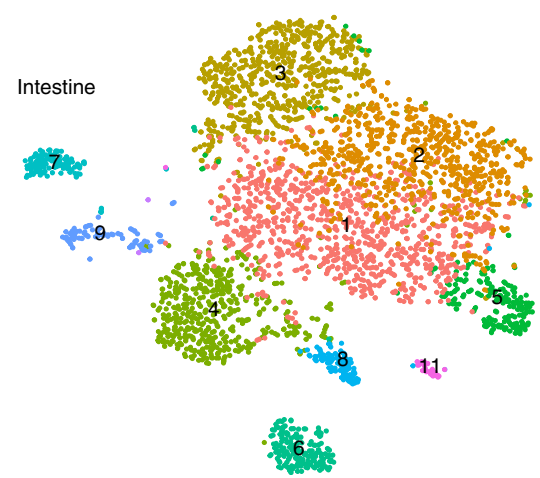

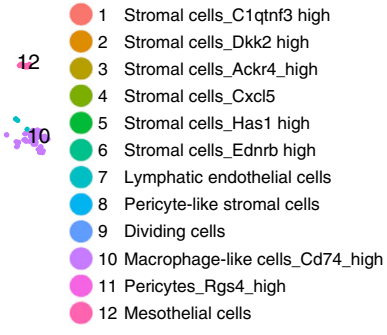

b

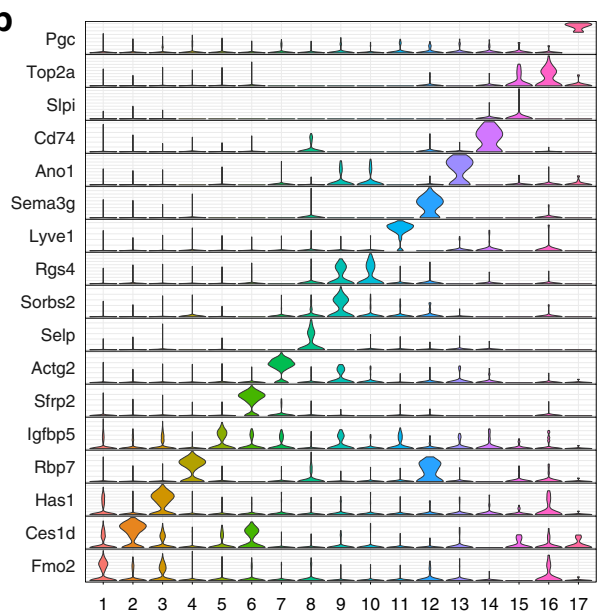

d

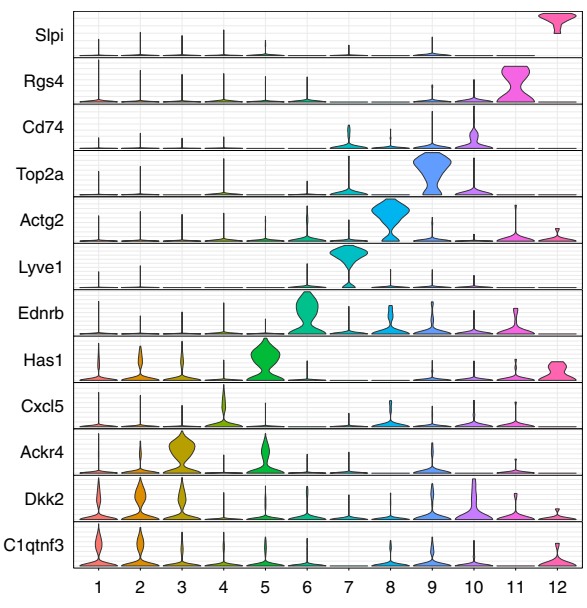

Intestine_C7 0.8

Intestine_C1 0.7

Intestine_C2

Intestine_C3

Intestine_C5

Intestine_C4

Intestine_C6

Intestine_C8

Intestine_C11

Intestine_C9

Intestine_C10

Intestine_C12

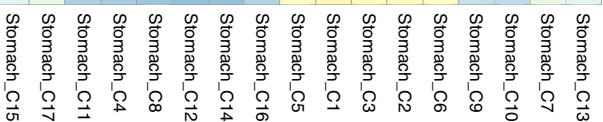

Stomach

f

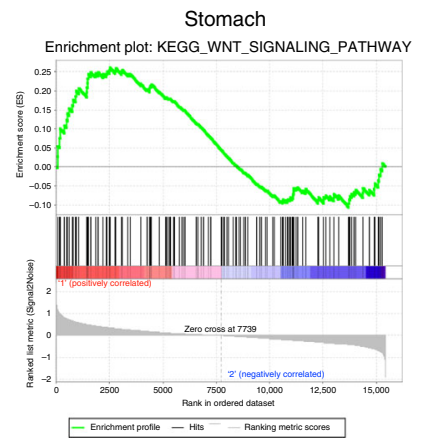

Intestine

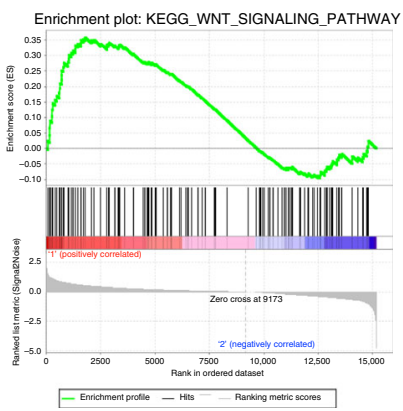

Fig. 1 Identification of conserved stromal cell populations between the stomach ad intestine. a, c t-SNE plots identify 17 different clusters in 4946 stomach cells (a) and 12 different clusters in 3459 intestinal cells (c). Cells from two mice were pulled for stomach (a) and intestinal (c) single cell RNA-seq. b, d Violin plots show expression levels of represented markers within each cluster in the stomach (b) and intestine (d). e Unsupervised hierarchical clustering shows gene expression correlation between the stomach and intestinal stromal cell clusters. A correlation of 0.7 was used to define stromal cell populations conserved between the stomach and intestine. $\mathbf{f}, \mathbf{g}$ The gene set enrichment analysis (GSEA) shows enrichment of the WNT signaling pathway in conserved populations compared to distinct populations.

populations also include telocytes (Fig. 2f, g). These cells also expressed Actg2 and its paralog, aSMA (Figs. 1b, d, 2h).

Wnt secretion by pericyte-like cells during regeneration. To investigate a role for pericyte-like cell populations as a gastrointestinal stem cell niche, we analyzed $\mathrm{Ng} 2-\mathrm{Cre}$; Rosa26 $6^{+/ m T m G}$ mice and found GFP-positive stromal cells marked by $\mathrm{Ng2-Cre}$ in close proximity to gastrointestinal epithelial cells surrounding the gland and crypt. In addition to the expression of Foxl1 ${ }^{21}$ (Fig. 2), immunogold transmission electron microscopy analysis demonstrated that these cells exhibit a long and very thin structure called a telopode (Fig. 3a; Supplementary Figs. 17 and 18). To examine the role of these pericyte-like cells as a source of Wnt niche signals, we performed smFISH in Ng2-Cre;Rosa26 ${ }^{+/ t d T o m a t o}$ mice and quantitative RT-PCR, and confirmed the expression of Wnt ligands such as Wnt $2 b$ and Wnt4 (Fig. 3b, c; Supplementary Figs. 19-21). To determine the significance of these Wnt secreting stromal cells as a stem cell niche, we conditionally deleted Wntless ( Wls), a transmembrane protein essential for the secretion of lipid modified 
a
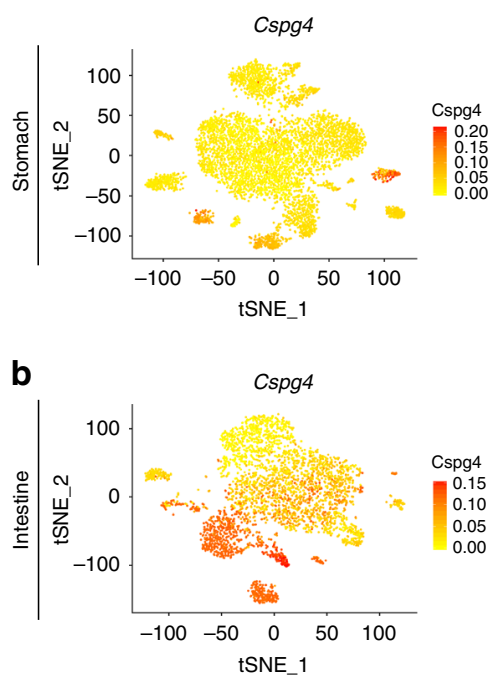

C

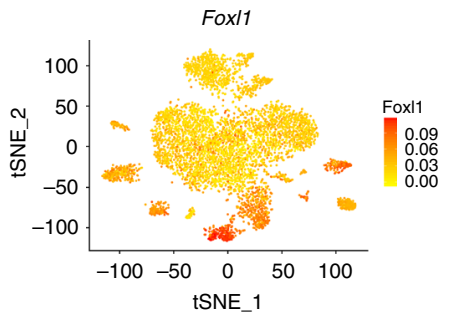

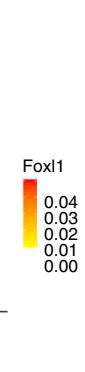

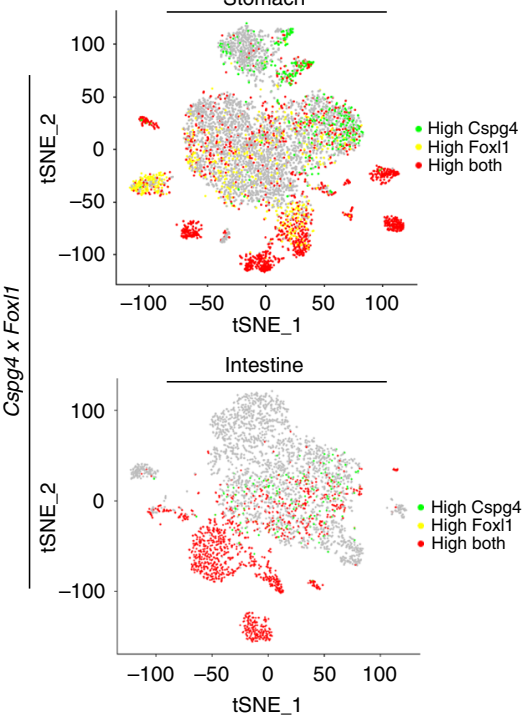

d

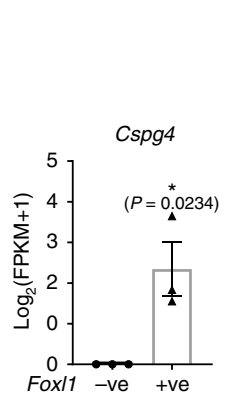

GSE94072

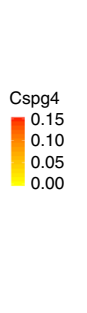

e

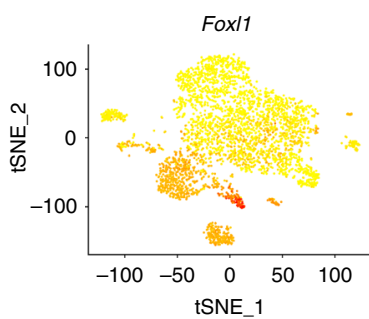

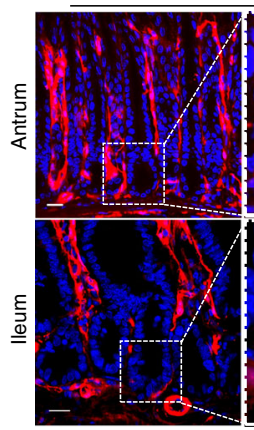

NG2DsRed/DAPI

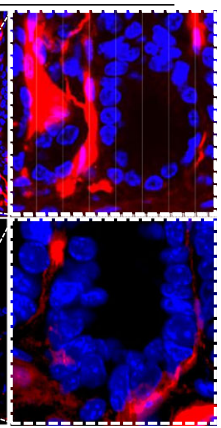

f

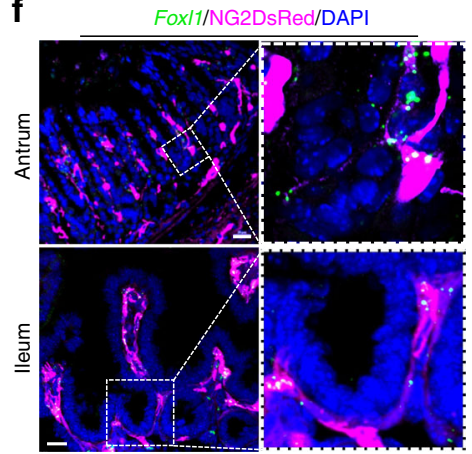

g

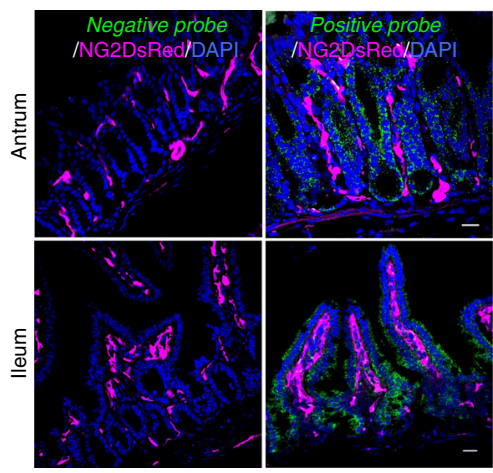

h
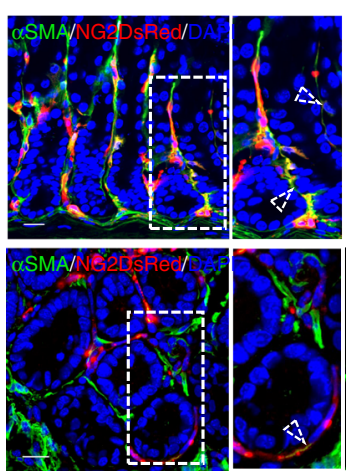
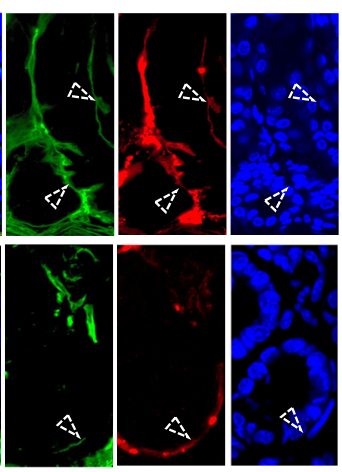

Fig. 2 Co-expression of pericyte and telocyte markers by conserved stromal cells. a, b Feature plot (FP) of Cspg4 (Ng2) and Foxl1 in stomach (a) and intestine (b). c co-FP of Cspg 4 and Fox/1 in stomach and intestine t-SNE plot. Red dots mean cells expressing both genes. $\mathbf{d}$ Comparison of Cspg4 expression levels between Fox17-positive and -negative cells from the GSE94072 dataset shows highly enriched expression of Cspg4 in Fox/1-positive cells. $n=3$, biologically independent samples. ${ }^{\star} P<0.05$ by unpaired student $t$ test. Values are mean \pm SEM. e Real-time Ng2 expression in antral glands and intestinal crypts of $\mathrm{Ng} 2^{+/ D s R e d}$ mice shows pericyte-like stromal cells in proximity to stomach and intestinal epithelial cells. Scale bars indicate $20 \mu \mathrm{m}$. $\mathbf{f}$ Singlemolecule FISH (smFISH) of Foxl7 (green puncta) in Ng2+/DsRed (magenta) mice. Scale bars indicate $20 \mu \mathrm{m}$. Area in dotted squares in left are magnified to right dotted square. $\mathbf{g}$ Images of negative (left) control with a probe targeting the DapB gene (accession \# EF191515) from the Bacillus subtilis strain SMY, a soil bacterium, and of positive (right) control with probes targeting Polr2a (green) and Ppib (blue) in the antrum (upper panel) and ileum (lower panel) related to Fig. 2f. Scale bars indicate $20 \mu \mathrm{m}$. h IF staining of $\alpha \mathrm{SMA}$ in $\mathrm{Ng} 2^{+/ D s R e d}$ mice. Empty arrowhead indicates co-expressing cells; filled arrowhead indicates only DsRed positive cells; arrow indicates green color single positive cells. Sub-population of $\alpha$ SMA expressing cells are co-labeled with DsRed. Scale bars indicate $20 \mu \mathrm{m}$. Blue is DAPI.

$\mathrm{Wnts}^{28}$. The isthmus of antral gastric glands, where Lgr5 is expressed has been also proposed as a potential Wnt niche $10,23,29,30$. Wnt signaling is known to promote intestinal secretory lineage differentiation ${ }^{31-33}$. Although $\mathrm{Ng} 2-\mathrm{Cre} ; \mathrm{Wls} / \mathrm{fl}$ mice exhibited a shortened gut length with reduced numbers of goblet cells and
Paneth cells, suggesting a reduced level of Wnt signaling, we observed no significant change in gastrointestinal epithelial proliferation (Fig. 3d, e; Supplementary Fig. 22). However, the numbers of both stomach and intestinal stem cells marked by OLFM4 and Lgr5 were significantly decreased (Fig. 3f, g). 
a

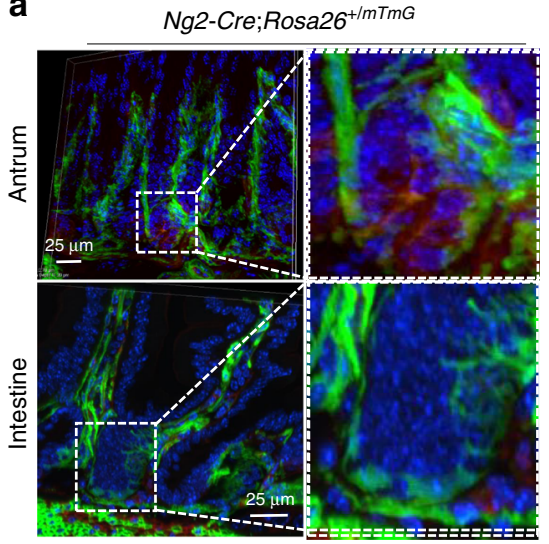

Ng2-Cre lineage tracing/DAPI b

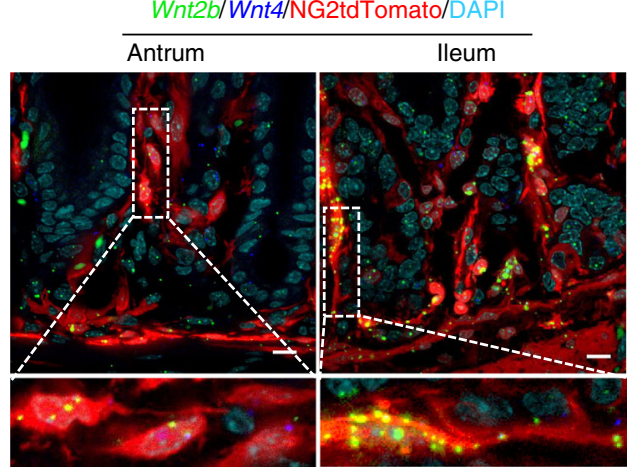

C

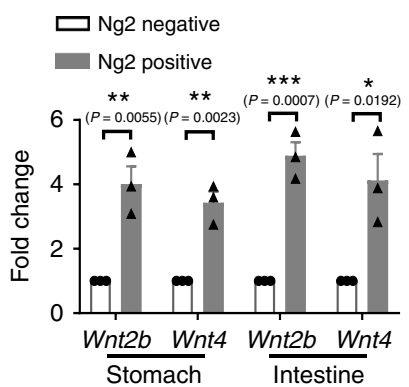

d

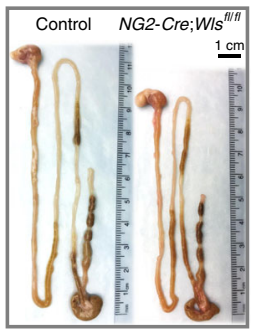

e

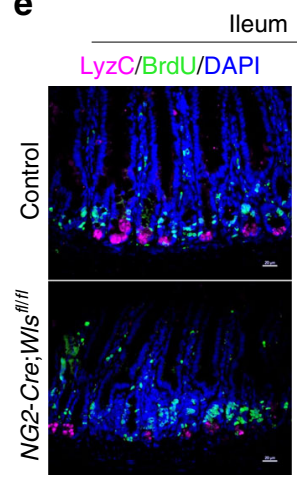

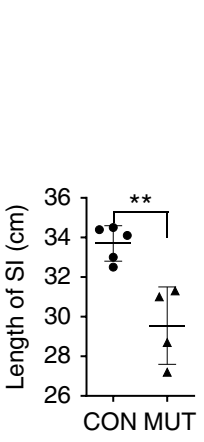

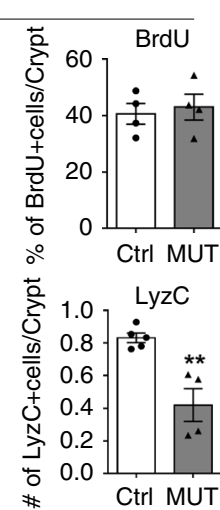

$\mathbf{f}$
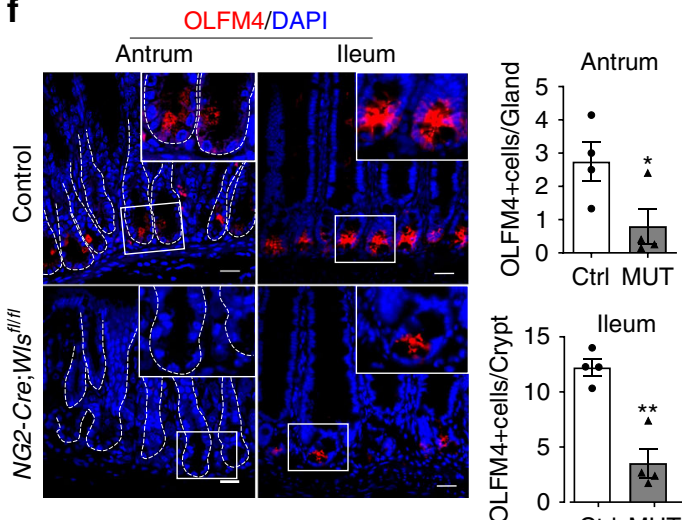

g

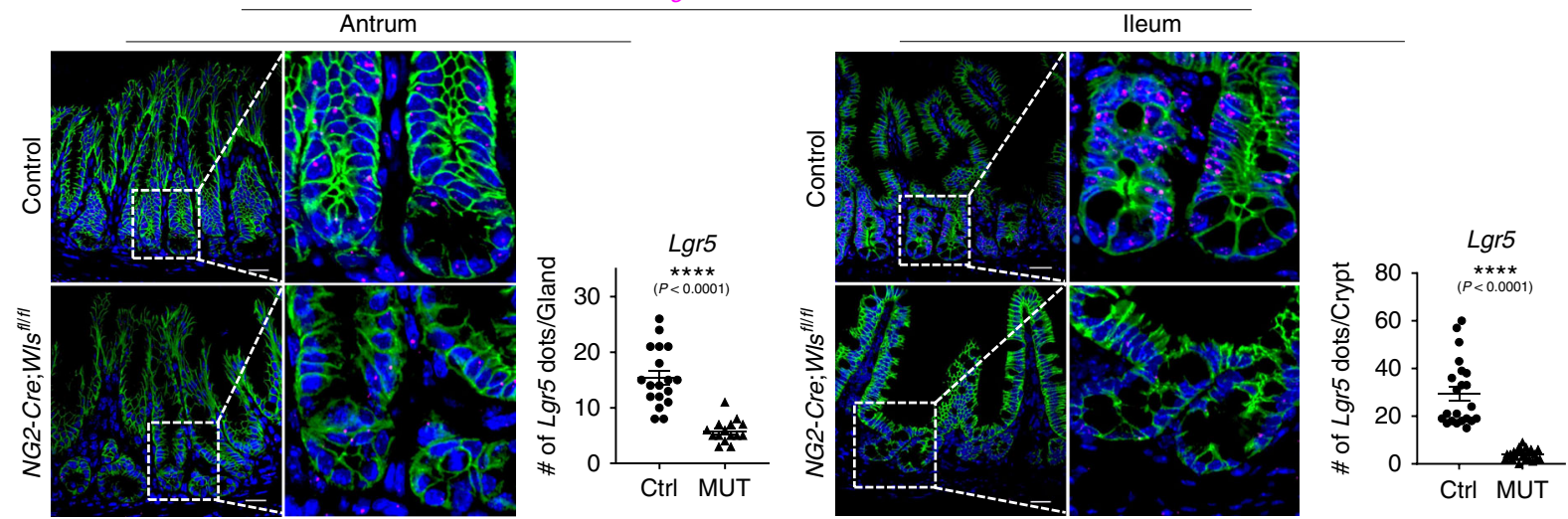

Fig. 3 Wnt secretion from pericyte-like stromal cells is required for adult stem cells homeostasis. a 3D images of pericyte-like stromal cells surrounding

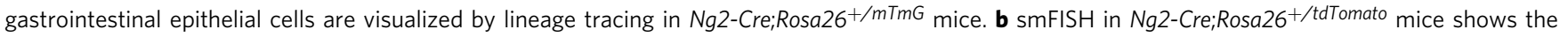
expression of Wnt2b (green puncta) and Wnt4 (blue puncta) in gastrointestinal pericyte-like stromal cells (tdTomato-red). c Quantitative RT-PCRs shows significantly increased levels of Wnt2b and Wnt4 expression in Ng2-tdTomato positive stomach and intestinal stromal cells compared to Ng2-tdTomato negative cells ( $n=3$ per group). d The intestinal length is significantly reduced in $\mathrm{Ng} 2$-Cre; $W / s f l / f l$ mice compared to the controls ( $n=5$ per group). e Immunofluorescence staining (IF) of BrdU and Lysozyme C (a Paneth cell marker) shows no defects in intestinal proliferation but a mildly decreased number of Paneth cells in $\mathrm{Ng} 2-\mathrm{Cre} ; \mathrm{WIs} / \mathrm{fl} / \mathrm{mice}$ compared to the controls ( $n=4$ per group). $\mathbf{f} \mathrm{IF}$ of OLFM4 in antrum and ileum shows significantly decreased numbers of OLFM4+ gastrointestinal stem cells in in $\mathrm{Ng} 2-\mathrm{Cre} ; \mathrm{WIs}{ }^{f / / f l}$ mice compared to the controls $(n=4$ per group). Scale bars indicate $20 \mu \mathrm{m} . \mathbf{g ~ s m F I S H}$ of $L g r 5$ in the antrum and ileum. Magenta dots indicate $L g r 5$ transcripts. The number of $L g r 5$ transcripts was significantly reduced in $\mathrm{Ng} 2-$ Cre; $W \mid s^{f l / f l}$ mice compared to the controls. Scale bars indicate $20 \mu \mathrm{m} .{ }^{\star} P<0.05,{ }^{\star \star} P<0.01,{ }^{\star \star \star} P<0.001,{ }^{\star \star \star \star} P<0.0001$. $P$ values were determined using nonparametric unpaired Student's $t$ test. Values are mean \pm SEM. Each $n$ means biologically independent animals and experiments. 
a

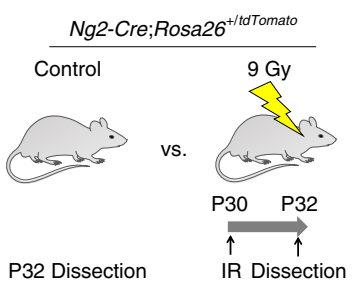

P32 Dissection b

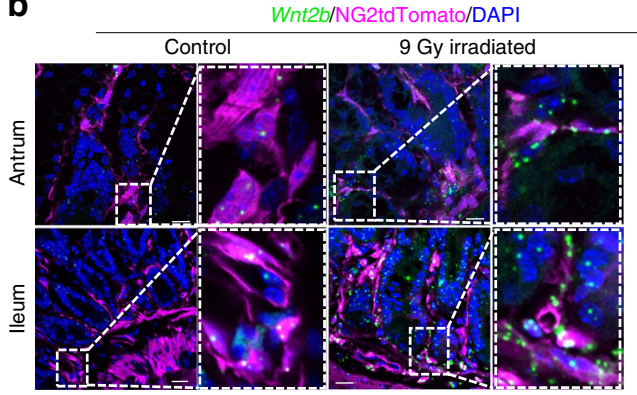

C

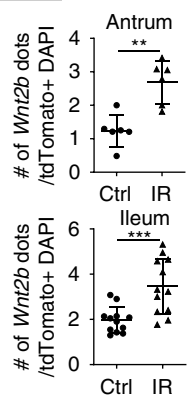

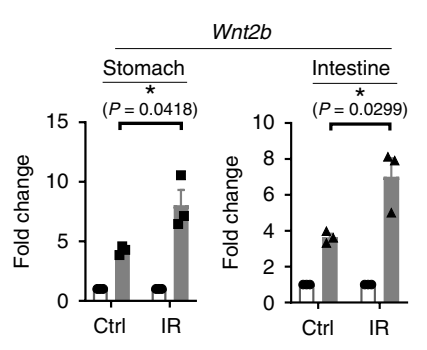

$\square$ Ng2 negative

Ng2 positive

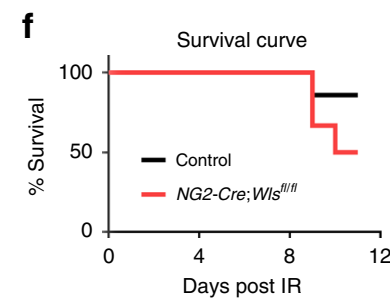

g

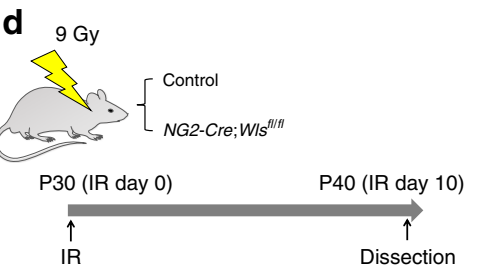

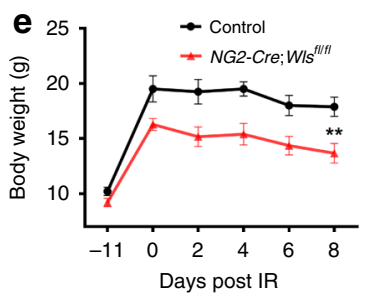
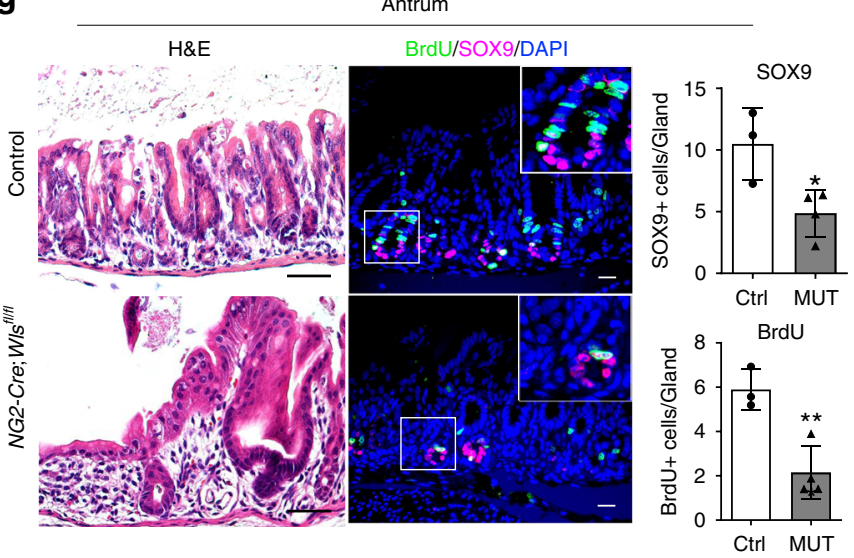

i

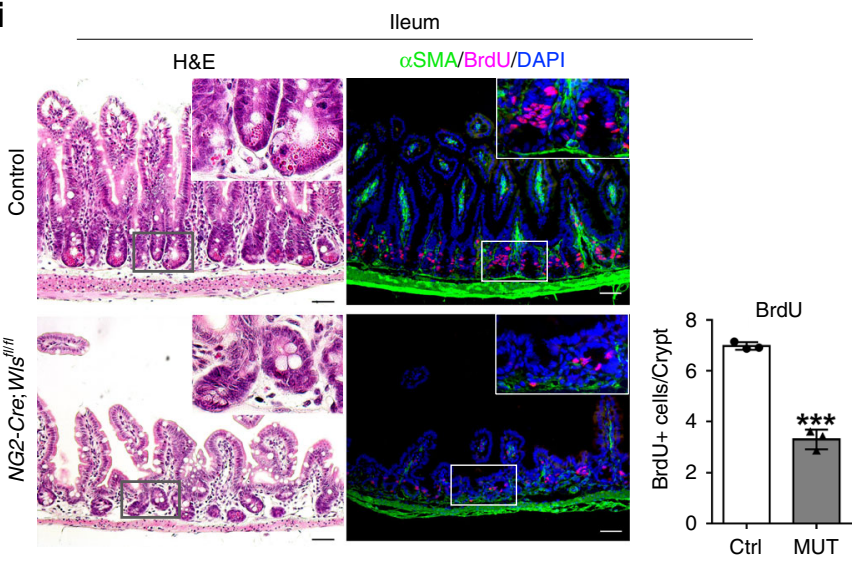

h

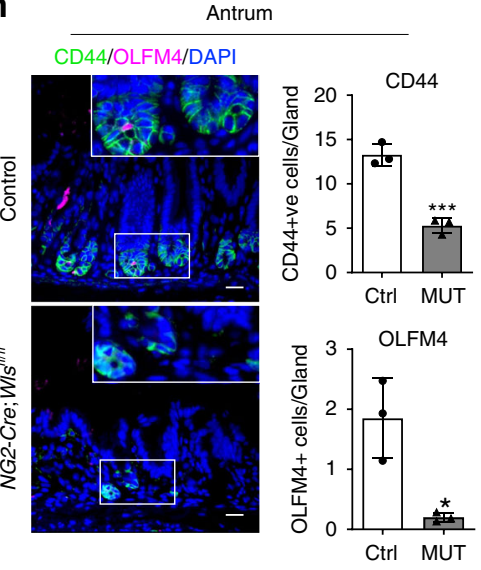

j

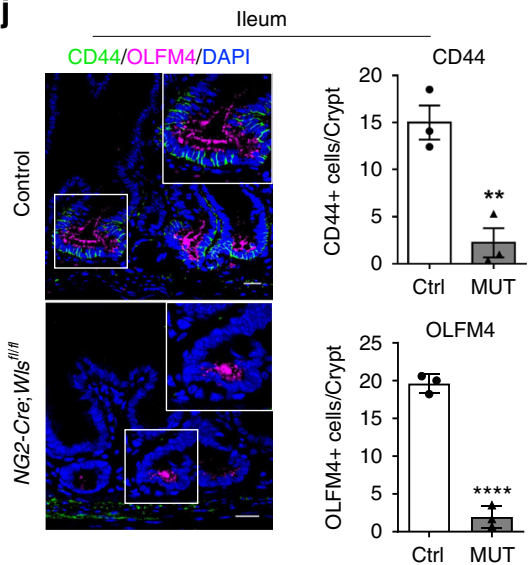

Despite the reduced numbers of stomach and intestinal stem cells in $\mathrm{Ng} 2-\mathrm{Cre} ; \mathrm{Wl} \mathrm{s}^{l / f l}$ mice, no proliferation defects were found (Fig. 3e; Supplementary Fig. 22), and they were able to breed properly, suggesting additional sources of niche signals. Supporting this notion, other stromal cell populations not labeled by Ng2-Cre expressed Wnt ligands (Supplementary Fig. 11). Given the proximity of the pericyte-like stromal cells to gastrointestinal epithelial stem cells, their expression of Wnt ligands and the role of Wnt signaling in stem cell renewal ${ }^{7}$, we hypothesized that Wnt secretion from these cells is critical during regeneration. To address this question, we irradiated Ng2-Cre;Rosa26+/tdTomato mice and examined the expression of Wnt ligands. Interestingly, we found significantly increased levels of $W n t 2 b$ expression in both the Ng2-labeled pericyte-like stromal cells (tdTomato+) cells and epithelial cells, suggesting a potential role for pericytelike stromal cells as a critical niche during regeneration (Fig. $4 \mathrm{a}-\mathrm{c}$ ). To address these stomach and intestinal stromaspecific roles in regeneration, we subjected both control and 
Fig. 4 Ng2 + pericyte-like stromal cells constitute a critical stem cell niche during regeneration. a Schematic diagram for whole body irradiation and dissection at postnatal day 32 (P32). b smFISH of Wnt2b (Green) in Ng2-Cre;Rosa26 /tdTomato mice $48 \mathrm{~h}$ after whole body irradiation (9 Gy) shows their significantly increased expression compared to the non-irradiation controls ( $n=3$ per group, 2-4 images per mice were counted for Wnt2b expression). c Quantitative RT-PCR shows increased levels of Wnt2b and Wnt4 expression in Ng2-Tdtomato positive cells after 9 Gy irradiation, compared to nonirradiated Ng2-tdTomato positive cells ( $n=3$ per group). $\mathbf{d}$ Timeline of whole body irradiation. e Body weight change of mice after irradiation ( $n=6$ per group). $\mathbf{f}$ Survival curve during 10 days after $9 \mathrm{~Gy}$ of irradiation ( $n=7$ per group). $\mathbf{g}$ H\&E staining (left), and IF of BrdU (green) and SOX9 (magenta) show a significantly decreased level of gastric epithelial proliferation (BrdU+ cells/gland) and decreased number of Sox9 progenitor cells (Sox9+ cells/gland) in antrum of $\mathrm{Ng} 2-\mathrm{Cre} ; \mathrm{WIs} f / / f l$ mice compared to the controls ( $n=3$ per group). $\mathbf{h}$ IF of CD44 (green) and OLFM4 (magenta) show a significantly decreased number of CD44+ and OLFM4+ progenitors and stem cells, respectively, in the antrum of $\mathrm{Ng2-Cre;WIs/ffl}$ mice compared to the controls $(n=3$ per group). $\mathbf{i}$ H\&E staining (left), and IF of BrdU (magenta) and $\alpha \mathrm{SMA}$ (green) show a significantly decreased number of BrdU+ cells in the ileum of $\mathrm{Ng} 2-\mathrm{Cre}$; WIs ${ }^{f l} / f \mid$ mice compared to the controls ( $n=3$ per group). $\mathbf{j}$ IF of CD44 (green) and OLFM4 (magenta) show a significantly decreased number of CD44+ and OLFM4+ progenitors and stem cells, respectively, in the ileum of $\mathrm{Ng} 2-\mathrm{Cre} ; \mathrm{Wls}{ }^{f l} / \mathrm{fl}$ mice compared to the controls ( $n=3-4$ per group). For all, ${ }^{\star} P<0.05$, ${ }^{\star \star} P<0.01,{ }^{\star \star \star} P<0.001,{ }^{\star \star \star} P<0.001,{ }^{\star \star \star \star} P<0.0001$. $P$ values were determined using nonparametric unpaired Student's $t$ test. Scale bars indicate $20 \mu \mathrm{m}$. Values are mean \pm SEM. Each $n$ means biologically independent animals and experiments.

Ng2-Cre;Wlstlfl mice to irradiation and analyzed them for regeneration 10 days later (Fig. $4 \mathrm{~d}$ ). When compared to the controls, the survival and proliferation of both stomach and intestinal regenerative progenitors and stem cells were compromised in Ng2-Cre; Wlstlfll mice, demonstrating the critical role of the pericyte-like stromal cells as a stem cell niche during regeneration (Fig. 4e-j). While Ng2-Cre lineage labeled pericryptal cells rarely proliferate in normal adult homeostasis, their proliferation was significantly increased after irradiation (Supplementary Fig. 23a). Consistent with this data, we also found that Ng2-Cre lineage labeled pericryptal cells are radioresistant, compared to epithelial cells (Supplementary Fig. 23b). Interestingly, it has been reported that the expression of Shh increases after irradiation and promotes $S h h$-mediated tumorigenesis in brain $^{34}$. Indeed, we found an increased level of its expression in epithelial cells (Supplementary Fig. 24). Although this result would likely be influenced by defective stem homeostasis found in $\mathrm{Ng2-Cre;} \mathrm{Wls} f / f l$ mice, it still demonstrates the requirement for pericyte-like stromal cells as a Wnt stem cell niche in regeneration.

Hh-Wnt signaling axis in pericyte-like stromal cells. We next investigated how these gastrointestinal stromal Wnt niche signals are regulated. It has recently been shown that intestinal pericryptal cells co-express Hh target and Wnt ligand genes, and that Hh-responsive cells constitute a key colonic stem cell niche ${ }^{17,22}$. Activated exclusively in the gastrointestinal mesenchyme ${ }^{35}, \mathrm{Hh}$ signaling is required for gut epithelial and stromal proliferation, as well as muscle differentiation ${ }^{36,37}$. Mice depleted of $\mathrm{Hh}$ signaling also show defects in intestinal stem cells ${ }^{36}$. Therefore, we analyzed $\mathrm{Hh}$ target genes in our single cell RNA-seq data and found that the stromal cell populations conserved between the stomach and intestine are highly enriched for the Hh pathway, compared to the distinct stromal cell populations (Fig. 5a). To further investigate the Hh regulation of Wnt signaling, we activated $\mathrm{Hh}$ signaling in the $\mathrm{Ng2-Cre} \mathrm{labeled} \mathrm{pericyte-like} \mathrm{stromal}$ cells and detected elevated levels of both stomach and intestinal epithelial cell proliferation (Fig. 5b, c). Corroborating this data, the numbers of gastric progenitors and stem cells positive for OLFM $4^{38}$, SOX $9^{39}$, and CD $44^{40}$ (Wnt target genes), were significantly increased (Fig. 5b; Supplementary Fig. 25). Consistent with the role of Wnt signaling in intestinal secretory lineage differentiation ${ }^{31-33}$, the number of goblet cells also was significantly increased upon $\mathrm{Hh}$ activation during development (Fig. 5d). Moreover, the smFISH analysis showed increased levels of gastrointestinal stromal Wnt ligand expression in these mice, demonstrating $\mathrm{Hh}$ activation of stromal stem cell niche signals (Fig. 5e, f; Supplementary Fig. 26).
GLI2 activation of Wnt ligands in conserved stromal cells. Although Hh signaling plays a critical role in many different organs and tissues, including the stomach and intestine, the instability of Hh downstream GLI transcriptional factors (TFs) has prevented the mapping of their genomic binding regions at a high resolution ${ }^{41}$. Consequently, understanding its transcriptional mechanisms has proven to be extremely challenging. To overcome this barrier, we utilized mice with conditional alleles of the key Hh-negative regulators, Sufu and Spop, which are known to act on GLI TFs, sequestering them in the cytoplasm and targeting them to proteasome degradation, respectively ${ }^{41}$. These mice were then crossed with mice carrying a Bap $x 1^{+/ C r e}$ allele to specifically delete these genes in the gastrointestinal mesench$\mathrm{yme}^{24}$ (Supplementary Fig. 27). Bap $x 1^{+/ C r e} ;$ Sufu $w^{f l f l} ;$ Spop $p^{f / f l}$ mice stabilized GLI TFs, thereby activating Hh signaling; these mice exhibited severe gastrointestinal defects with abnormal proliferation in both epithelial and mesenchymal tissues (Supplementary Fig. 28). Taking advantage of the stabilized key $\mathrm{Hh}$ transcription factor involved in gut development, GLI2 ${ }^{36}$, we mapped its genomic binding regions in the intestine, demonstrating direct transcriptional activation of intestinal stromal Wnt ligands by GLI2 $2^{42}$. Since signaling and transcriptional mechanisms of stomach stromal Wnt niche signals remained unknown,

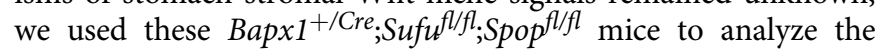
stomach and compared with the intestinal data. Gene network analysis of the transcriptomes generated from these Sufu and Spop double knockout (DKO) gastrointestinal tissues showed common enrichment in muscle development and the Wnt pathway (Fig. 6a).

To determine if these $\mathrm{Hh}$ target genes are activated via super enhancers, we performed ChIP-Seq for H3K27ac marks with the Sufu and Spop DKO gastrointestinal tissues and screened for super enhancers by using the Rank Ordering of Super-Enhancers (ROSE) program ${ }^{43,44}$. We found a surprisingly high number (538) of Hh activated super enhancers conserved between the stomach and intestine (Fig. 6b, c). Organ-specific enhancers were also revealed: Stomach- and intestine-specific super enhancers were identified near the organ-specific $\mathrm{Hoxc}^{45}$ and $\mathrm{Nr} 2 \mathrm{fl}^{46}$ loci, respectively (Fig. $6 \mathrm{~d})^{47}$. Moreover, our mapping of genome-wide binding sites of GLI2 identified more than one thousand GLI2 peaks conserved between the stomach and intestine (Fig. 6e). The KEGG pathway analysis of these overlapping peaks demonstrated significant enrichment for the Hh and Wnt pathways (Fig. 6f). These peaks were identified in $\mathrm{H} 3 \mathrm{~K} 27 \mathrm{ac}$ marked enhancer regions conserved between the stomach and intestine, near the Wnt and Hh pathway genes (Wnt2b, Wnt9a, and Ptc1) (Fig. 6g). To validate direct transcriptional regulation by GLI2 in gastrointestinal mesenchymal cells, we were able to culture primary stomach and intestinal mesenchymal cells. We then cloned GLI2-bound 
a
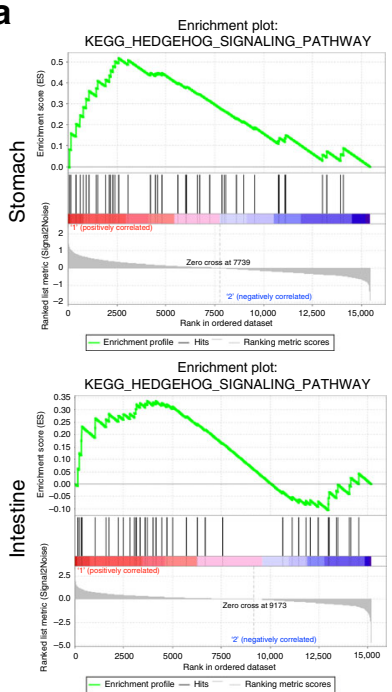

b
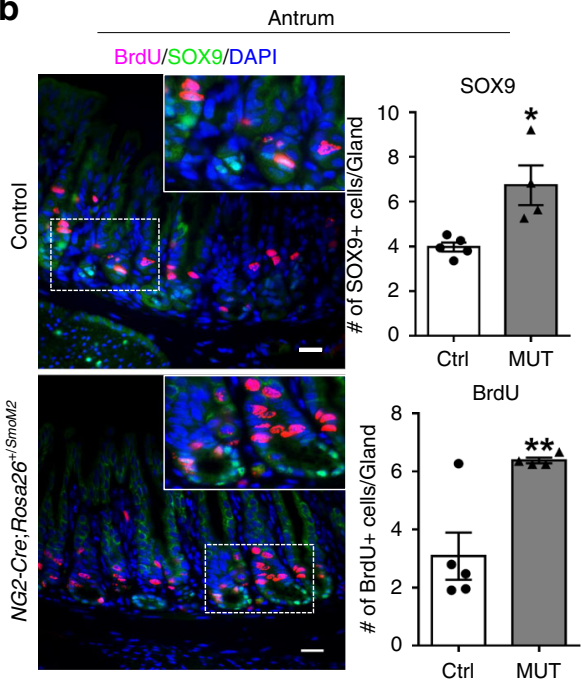

C

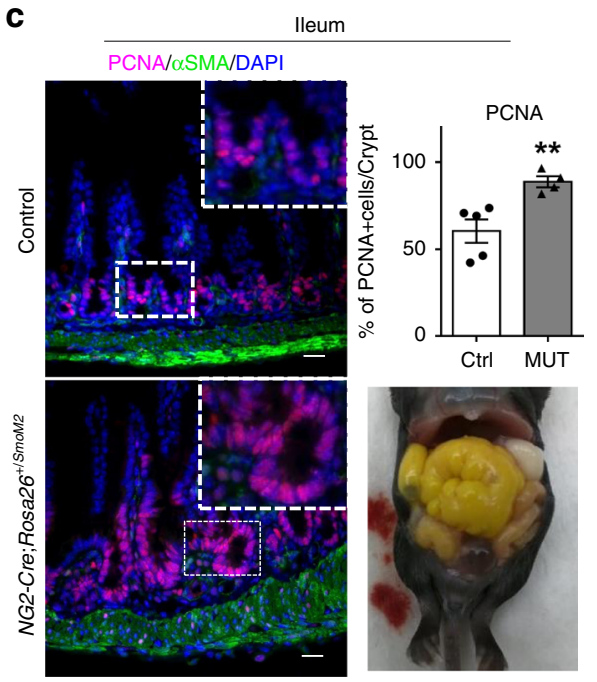

d

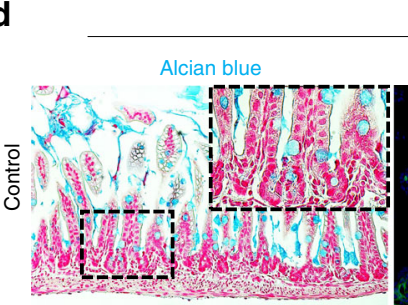

Ileum
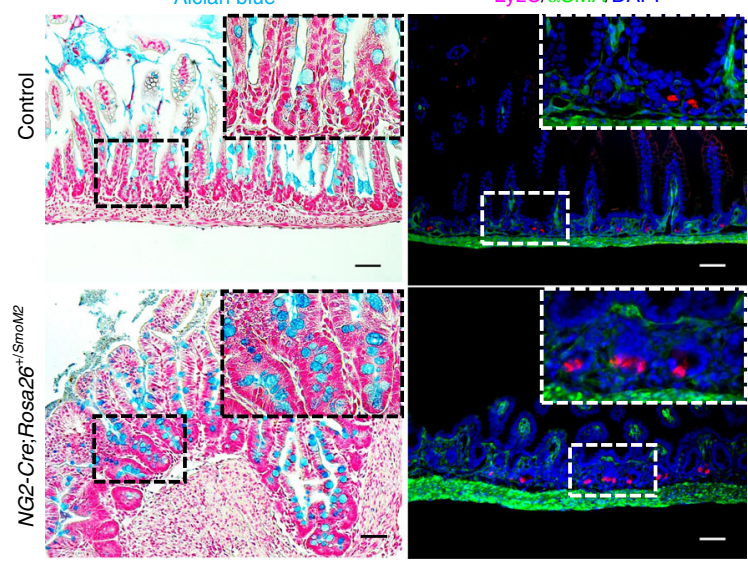

yzC/ $\alpha$ SMA/DAP
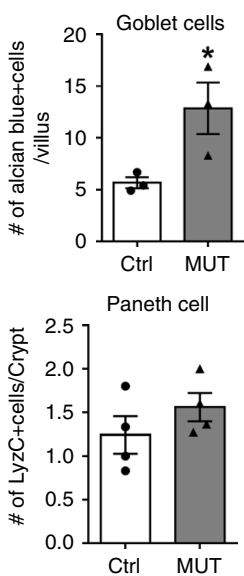

e

Antrum

$\mathbf{f}$

Ileum
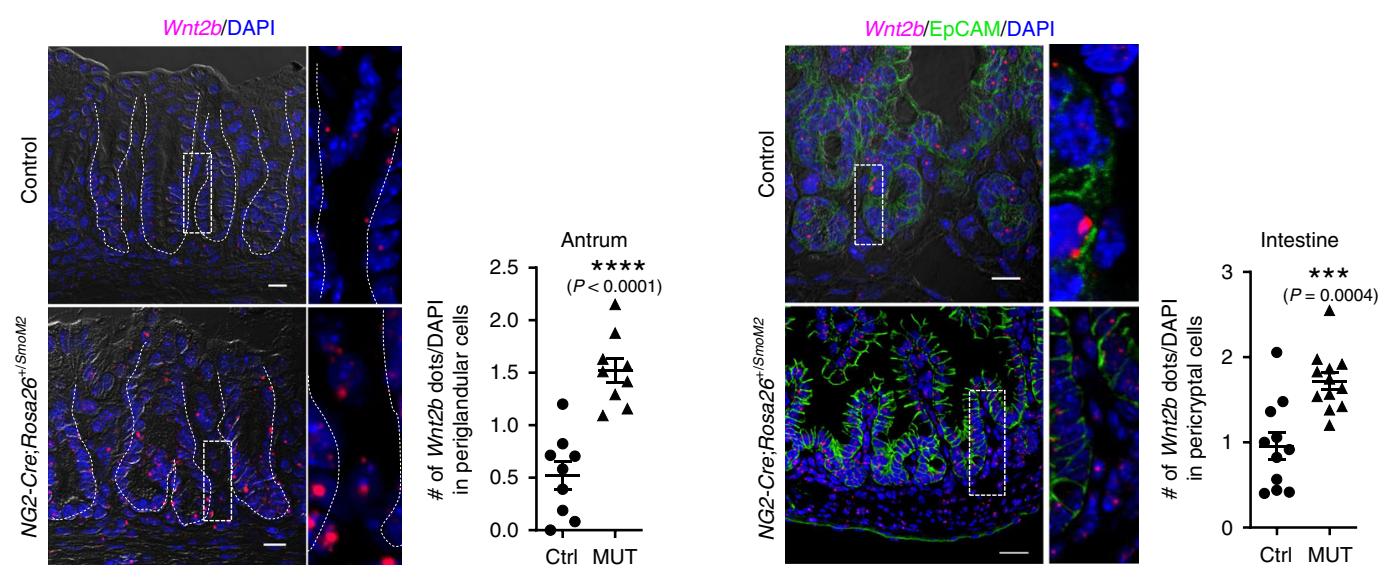

regions near $W n t 2 b$ and $W n t 9 a$ loci into plasmids containing a luciferase reporter gene and performed a transcriptional reporter assay upon co-expression of Gli2. We confirmed GLI2 activation of these reporter genes in gastrointestinal mesenchymal cells (Fig. 6h, i; Supplementary Fig. 29). Corroborating this data, the expression of Gli2 was enriched in $\mathrm{Ng} 2$-Cre labeled and Cspg4 $(\mathrm{Ng} 2)$ expressing pericyte-like stromal cells (Supplementary
Fig. 20, 30). Together, this work reveals that the GLI2-mediated transcriptional activation of stromal Wnt niche signals, and this direct regulation in gut stromal cells is conserved between the stomach and intestine.

Redundant role of stromal Wnt secretion in gut development. Although this study has revealed conserved signaling mechanisms 
Fig. 5 Hh signaling in pericyte-like stromal cells activates epithelial Wnt signaling. a GSEA plot demonstrates enrichment of the Hh signaling pathway in conserved pericyte-like gastrointestinal stromal cells compared to distinct populations. b IF of BrdU and SOX9 in the stomach antrum. The number of SOX9+ and BrdU+ stomach progenitors are significantly increased in Ng2-Cre;Rosa26+/SmoM2 mice compared to the controls ( $n=3$ per group). c IF of PCNA and $\alpha$ SMA in the intestine. The number of PCNA+ progenitors is significantly increased in Ng2-Cre; Rosa26 $6^{+/ S m o M 2}$ mice compared to the controls ( $n=3$ per group), ${ }^{\star} P<0.05$. Mutant pups show a dilated small intestine (Right bottom panel). d IF of Lysozyme $C$ (LyzC, right panel) and Alcian blue staining (left panel) shows increased numbers of intestinal Paneth cells and goblet cells, respectively, in $\mathrm{Ng} 2-\mathrm{Cre} ; \mathrm{Rosa} 26^{+/ S m o M 2}$ mice compared to the controls. ${ }^{\star} P<0.05$. Scale bars indicate $50 \mu \mathrm{m}$. e, $\mathbf{f}$ smFISH combined with differential interference contrast (DIC) imaging demonstrates significantly increased levels of Wnt2b expression (magenta puncta) in gastrointestinal pericyte-like stromal cells of $\mathrm{Ng} 2-\mathrm{Cre} ; \mathrm{Rosa}_{2} 6^{+/ \mathrm{SmoM} 2}$ mice compared to the controls ( $n=3$ per group). Scale bars indicate $20 \mu \mathrm{m},{ }^{\star} P<0.05,{ }^{\star \star} P<0.01,{ }^{\star \star \star} P<0.001,{ }^{\star \star \star \star} P<0.0001$. $P$ values were determined using nonparametric unpaired Student's $t$ test. Values are mean \pm SEM. Each $n$ means biologically independent animals and experiments.

of gastrointestinal stromal Wnt ligands, genetic inhibition of Wnt secretion in $\mathrm{Ng} 2$-expressing cells and their descendants resulted in only mild defects (Fig. 3d-g; Supplementary Fig. 22). Therefore, the significance of gastrointestinal stromal Wnt niche signals during development is still unclear. In fact, we found that Wnt ligands and/or agonizts are expressed in different gut stromal cell populations (Supplementary Fig. 11). To further define the difference between Bapx1 $1^{+/ C r e}$ and $\mathrm{Ng} 2$-Cre lineage labeled stromal cells, we first analyzed the percentage of tdTomato positive cells sorted from Bapx ${ }^{+/ C r e} ;$ Rosa $26^{+/ t d T o m a t o}$ and Ng2-Cre;Rosa26 $6^{+/ t d T o m a t o}$ mice and found that Bapx+/Cre labels significantly more stromal populations than Ng2-Cre (Supplemental Fig. 31). We then performed mass cytometry (CyTOF) of stomach and intestinal stromal cells isolated from $\mathrm{Ng} 2-\mathrm{Cre}$; Rosa $26^{+/ t d T o m a t o}$ and Bapx $1^{+/ C r e} ;$ Rosa $26^{+/ t d T o m a t o}$ mice for gut stromal cell type-specific markers. This analysis revealed that compared to Bapx $1^{+/ C r e}$, $\mathrm{Ng} 2$-Cre labels significantly fewer CD31-positive endothelial cells and Ly6c-positive stromal cells (Supplementary Figs. 32-34). Interestingly, these Ly6c positive cells failed to express a hematopoietic cell marker, CD45, representing unique stromal cell populations expressing Ly6c (Supplementary Figs. 35 and 36). Corroborating this data, our analysis of Bapx1 $1^{+/ C r e}$;Rosa $26^{+/ t d T o m a t o}$ mice showed that LYVE1positive lymphatic endothelial cells and PDPN-positive mesothelial cells expressed Wnt ligands and agonizts (Supplementary Figs. 11, 37, and 38). Taken together, these observations suggest a potentially redundant role for gastrointestinal stromal cells as a Wnt stem cell niche. Interestingly, our comparative analysis of single cell transcriptomes with available human gastrointestinal disease genes, identified by genome-wide association studies (GWAS), showed association of intestinal lymphatic endothelial and mesothelial cells with colon cancer and inflammatory bowel disease, also implying their potential roles in disease pathogenesis (Supplementary Fig. 39) ${ }^{48}$.

To address this potentially redundant role for Wnt-secreting gastrointestinal stromal cells, we used Bap $x 1^{+/ C r e}$ mice to more broadly target gastrointestinal stromal cells (Supplementary Figs. $1 \mathrm{a}$ and 31$)$. Bap $x 1^{+/ C r e} ; W l s^{f l / f l}$ mice exhibited remarkably severe phenotypes throughout the gastrointestinal tract (Fig. 7a-k; Supplementary Fig. 40). Consistent with the observation of Wnt signaling activation in the mouse forestomach, epithelial tissue during development ${ }^{4}, B a p \times 1^{+/ C r e} ; W l s f^{f l f l}$ mice showed a significant decrease in the size of the forestomach accompanied by reduced levels of epithelial proliferation, while properly maintaining a stomach-intestine boundary (Fig. $7 \mathrm{a}-\mathrm{d}$ ). In addition, the intestinal lumen was severely distended, and the intestine length was dramatically reduced; hematoxylin-eosin ( $\mathrm{H} \& \mathrm{E})$ staining also revealed epithelial depletion, with significantly reduced numbers of villi (Fig. $7 \mathrm{~g}, \mathrm{~h}$ ). Since this phenotype is morphologically similar to the gastric transformation defects found in $C d x 2$ knockout intestines ${ }^{49}$, we also analyzed gastric markers and CDX2 by IHC but found no transformation (Fig. 7b-d; Supplementary Fig. 40a). To analyze stem cell defects, we performed smFISH for Lgr5 and found its significantly reduced expression in both the stomach and intestine, demonstrating severe defects in developing stem cells (Fig. 7e, i; Supplementary Fig. 41). To measure epithelial Wnt signaling activity, we also analyzed the expression of known Wnt target genes such as SOX9 and CD44, and found a dramatic reduction of these markers (Fig. 7f, j; Supplementary Fig. 40b). Consistently, epithelial proliferation and secretory cell differentiation, both of which depend on Wnt signaling, were severely impaired (Fig. 7k; Supplementary Fig. 40c, d). These data demonstrate the essential role of gastrointestinal stromal Wnt niche signals during development.

\section{Discussion}

Wnt signaling had been thought to be restricted to the hindgut and critical for its specification ${ }^{6}$. Indeed, its abnormal activation in the stomach through the deletion of the stomach-specific mesenchymal factor, Barx1, led to partial intestinal transformation $^{4}$. In addition, the intestinal stem cell marker, Lgr5, was first identified by analyzing Wnt target genes ${ }^{50}$. Interestingly, Lgr5 was also expressed in a subpopulation of the antrum ${ }^{29,30}$; Wnt reporter expression was found to be temporarily active during stomach development, becoming later restricted to the distal stomach region, suggesting its temporal and regional roles ${ }^{4,23}$. Corroborating these data, recent studies have revealed a critical role for Wnt signaling in stomach development and stem cells ${ }^{9}$. In the intestine, Wnt ligands are expressed by Paneth cells surrounding stem cells ${ }^{11}$, and a number of studies have shown that mesenchymal cells also constitute a critical source of Wnt ligands $11,15,16,18-20,22$. However, neither the source of Wnt niche signals, nor how they are produced in the stomach was known. The stomach has no Paneth cells that would serve as source for Wnt ligands, implying that mesenchymal cells are a major Wnt stem cell niche. Here, our single cell and mouse genetic analyses demonstrate for the first time that stomach stromal cells constitute a critical Wnt stem cell niche during development and regeneration. We also demonstrate that the Hh downstream transcription factor, GLI2, directly induces the expression of Wnt ligands in the stomach. Since our single cell analysis was performed with stromal cells isolated from the glandular hindstomach, which includes both the corpus and the antrum, separate single-cell analysis would enable further definition of the stomach region-specific stromal niches.

Despite the fact that tissue cross-talk is known to provide tissue-specific niche signals to properly specify distinct organs ${ }^{1,2}$, our work not only identifies stromal cell populations that are highly conserved between the stomach and intestine, but also reveals a conserved mechanism by which $\mathrm{Hh}$ signaling induces stromal Wnt niche signals. A recent study has shown that stromal Hh-GLI2 provides key niche signals, which include both Wnt $2 b$ and Wnt2, to the mammary epithelial stem cells ${ }^{51}$. However, how these niche signals are produced by GLI2 was unknown. In addition to our earlier work in the intestine ${ }^{42}$, our current work in the stomach shows direct transcriptional activation by GLI2 of 


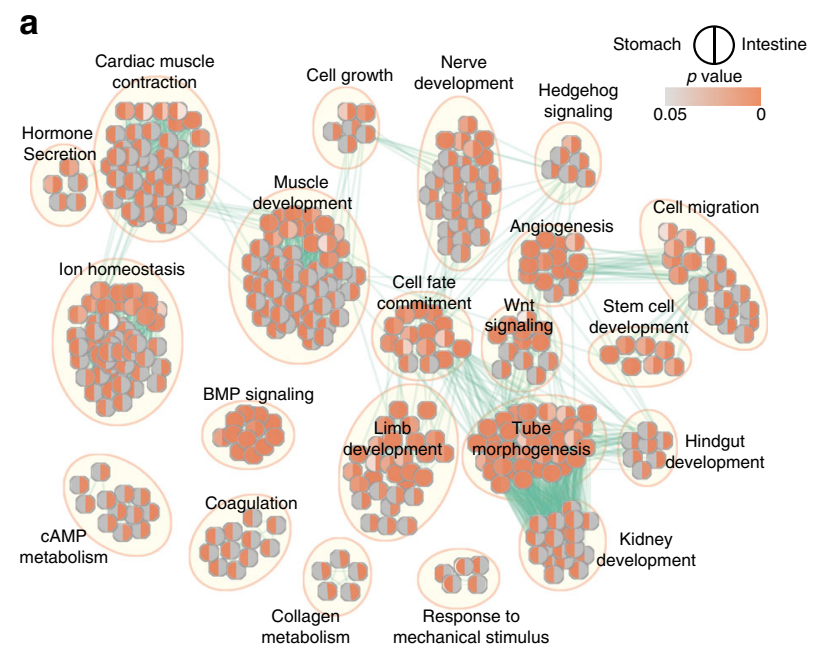

b

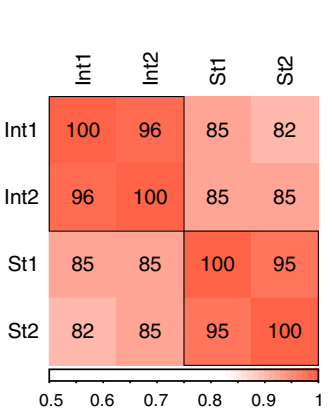

c

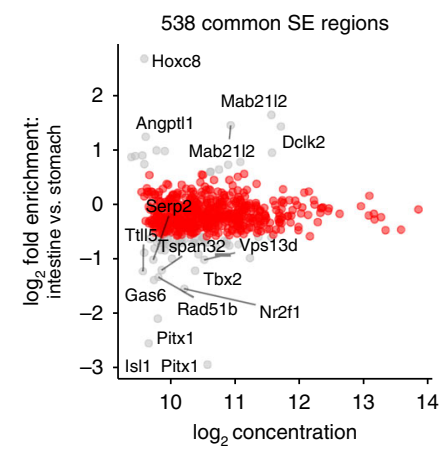

d

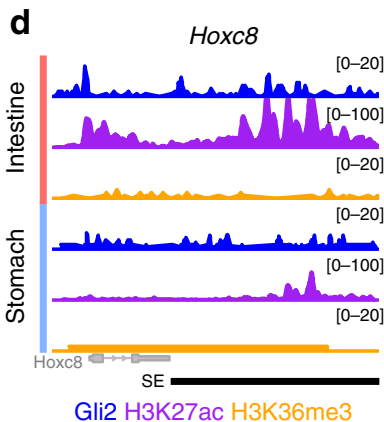

g

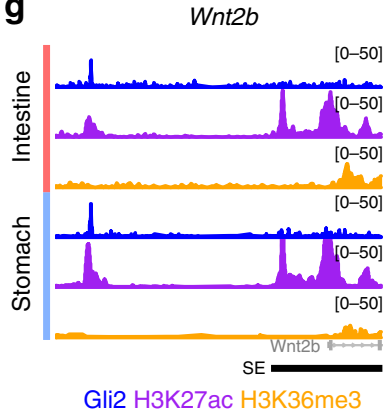

h

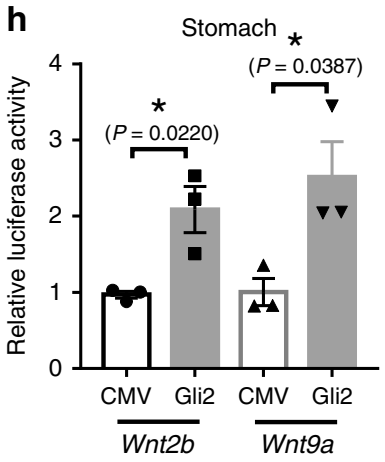

Nr2f1
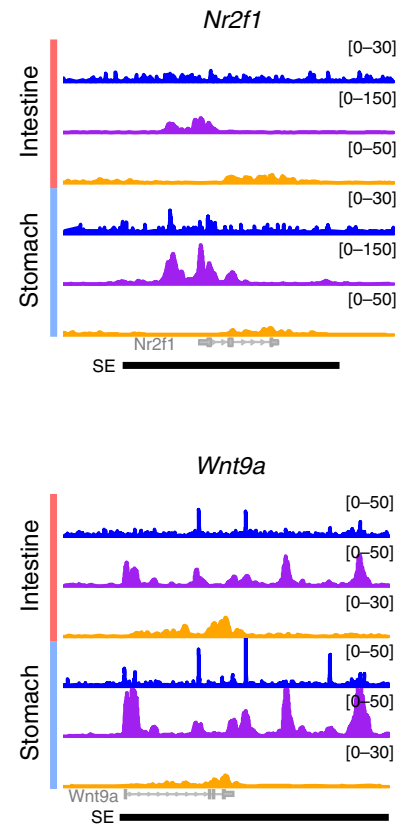

e

Gli2 ChIP peaks

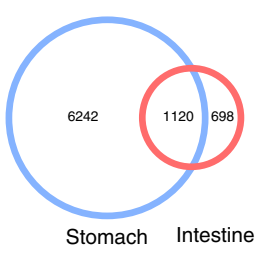

f

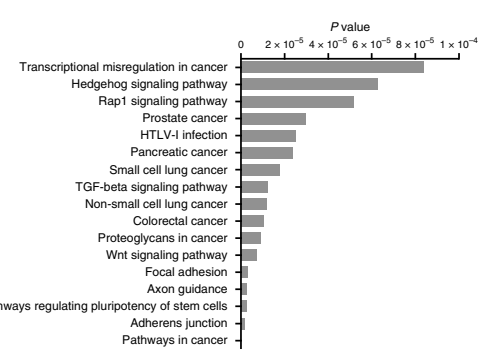

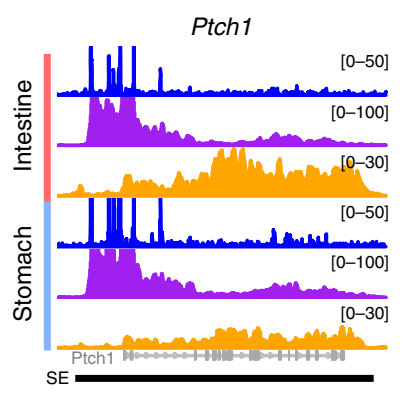

\section{i}

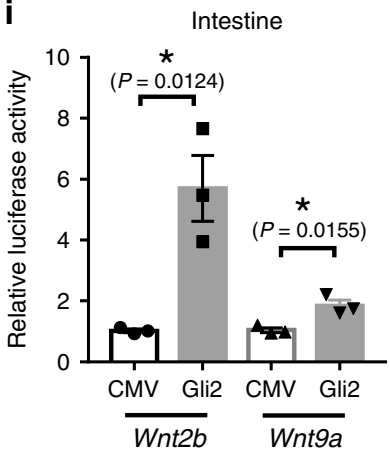

Wnt ligands such as Wnt2b and Wnt9a, further supporting $\mathrm{Hh}$ and GLI2-mediated direct activation of Wnt ligands as a conserved mechanism responsible for producing stromal stem cell niche signals. Mesenchymal Hh signaling is known to promote different types of cancer ${ }^{52}$. Our proposed Hh-GLI2 activation of stromal stem cell niche signals may also contribute to tumorignesis in different types of cancer.
Interestingly, genetic inhibition of Wnt secretion in $\mathrm{Ng} 2-$ derived cells, which include telocytes, led to much milder stem cell defects than the broad range of striking stem cell arrest defects found by Shoshkes-Carmel et al. Our work differs from these studies in that we utilized mice with the $\mathrm{Ng} 2$-Cre allele, which is expressed throughout embryonic development, in contrast to their inducible inhibition of Wnt secretion at the adult 
Fig. 6 Conserved GLI2 activation of Wnt niche signals between the stomach and intestine. a Enrichment network. Functional profiling of genes upregulated > twofold in DKO stomach and intestine vs. control. Left half circle: significance of gene set enrichment in stomach. Right half circle: significance of gene set enrichment in intestine. Analysis revealed that the majority of pathways are commonly enriched in both mutant stomach and intestine. Key genes sets: Hh signaling, Wnt signaling, BMP signaling, stem cell development, muscle development, cell fate commitment, angiogenesis, hindgut development (intestine only), response to mechanical stimulus (stomach only). b Correlation heatmap of H3K27ac ChIP-Seq signal at all SE regions identified from DKO stomach and intestine. Plot shows high within-group correlation, and relatively high inter-group correlation, indicating overlapping SEs in the two tissue types. c MA plot of H3K27ac ChIP-Seq signal at SE regions in DKO stomach and intestine confirmed findings in the correlation heatmap. Totally, 538 SE regions were common (fold-enrichment between the two tissues $<1.5$ ). Sixteen regions, mapped to 14 unique genes were tissue-specific (fold enrichment > 2). d Signals from GLI2, H2K27ac, and H3K36me3 ChIP-Seq experiments show examples of intestine (Hoxc8)- and stomach (Nr2f1)-specific regions regulated by GLI2. e Gli2 ChIP-Seq in DKO stomach and intestine identified over 1100 overlapping regions. f KEGG pathway analysis performed on genes associated with the 1120 overlapping Gli2 peaks. Significant enrichment was identified in Hh, TGF-beta, and Wnt signaling pathways. g Signals from GLI2, H2K27ac, and H3K36me3 ChIP-Seq experiments show examples of Hh signaling (Ptch1) and Wnt ligand (Wnt2b, Wnt9a) genes regulated by GLI2. All genes are within SE regions, and are bound by GLI2. h, i Validation of GLI2 activation of luciferase reporter fragments generated from GLI2 peak regions near (h) Wnt2b and (i) Wnt9a loci. $n=3$, Values are mean \pm SEM. ${ }^{\star} P<0.05$. $P$ values were determined using nonparametric unpaired Student's $t$ test. Each $\mathrm{n}$ means biologically independent animals and experiments.

stage using the Foxl1-CreERT2 allele. During development, these telocytes and other stromal cells might have adapted to produce compensatory stem cell niche signals to prevent drastic stem cell failure. Supporting the redundancy of stromal stem cell niches, we showed that Wnt ligands are expressed in different cell types throughout the mesenchyme (Supplementary Figs. 7 and 11). We also demonstrated that inhibition of Wnt secretion in stromal cells broadly leads to severe stem cell and developmental defects (Fig. 7). Although our Ng2-Cre lineage tracing showed ubiquitous labeling throughout the proposed telocyte region in proximity to gastrointestinal epithelial cells (Fig. 2e), and single cell analysis shows a significant overlap between $\mathrm{Ng} 2$ and Foxl1 expression (Fig. 2a-c), another possibility is that Foxl1 also labels rare but critical stromal cells not targeted by $\mathrm{Ng} 2$-Cre lineage tracing. Of note, a recent study has shown striking resistance of intestinal stromal cells to tamoxifen inducible Cre activation ${ }^{53}$. This data suggests that telocytes among intestinal stromal cells might have lost their resistance during evolution. While further investigation would be needed to better understand this cell population, genetic inhibition of Wnt secretion in Bapx $1^{\mathrm{Cre}}$-derived cells have demonstrated the significance of redundancy among different gastrointestinal stromal cell populations secreting stem cell niche signals. Although both $\mathrm{Bap} \times 1^{+/ C r e}$ and $\mathrm{Ng} 2$-Cre lineage-derived cells labeled telocytes, our single cell RNA-Seq and CyTOF analyses highlighted their difference. For example, many more CD31-positive endothelial cells and Ly6c positive stromal cells were represented in $\mathrm{Bap} \times 1^{\mathrm{Cre}}$-derived cells, compared to $\mathrm{Ng} 2$-Cre lineage labeled cells (Supplementary Figs. 32-36). Importantly, $L y 6 c$ is highly expressed in $\mathrm{Hh}$ signaling responsive colonic stromal cell populations that constitute a critical stem cell niche, further supporting their potential redundancy as an intestinal stem cell niche ${ }^{22}$. The identity of this stromal population would require further investigations.

Taken together, our study has revealed not only the conserved signaling and transcriptional mechanisms of stomach and intestinal stromal stem cell niches but also unexpected redundancy among stromal cell populations. Interestingly, telocytes have been found in many different mammalian organs ${ }^{54}$. Therefore, it would be useful to determine if $\mathrm{Hh}$ and GLI2-mediated activation of Wnt ligands is also conserved in the telocytes of other organs.

\footnotetext{
Methods

Mouse strains. Mice were housed in specific pathogen free barrier facilities. Mice handling and all experiments performed were monitored in accordance with protocols approved by The Centre for Phenogenomics (TCP) Animal Care Committee. Bapx $1^{\text {Cre }}$ mice were obtained as a gift from Dr. Warren Zimmer. A Spopfl allele was obtained from the trans-NIH knock-out mouse project (KOMP) Repository. Ng2-Cre, Ng2 ${ }^{\text {DsRed }}$, Myh11-CreERT2, Rosa26 ${ }^{m T m G}$, Rosa26 $6^{\text {tdTomato }}$ Rosa26 ${ }^{\text {SmoM2 }}$, and $W l s t$ mice were purchased from The Jackson Laboratory.
}

Embryos were dissected at E17.5 or E18.5; mice 2-5 weeks of age were used for analyses. Cre recombinase was activated by gavage of $1 \mathrm{mg}$ tamoxifen (Invitrogen) or by intraperitoneal injection of $1 \mathrm{mg}$ tamoxifen for 2 or 3 days. Tamoxifen was dissolved in corn-oil (Sigma) as a stock concentration of $10 \mathrm{mg} / \mathrm{ml}$. BrdU (BD Biosciences, $50 \mathrm{mg} / \mathrm{kg}$ ) was injected intraperitoneally in mice, and they were sacrificed $1 \mathrm{~h}$ later.

Stromal cell isolation for single-cell RNA sequencing. Single-cell sequencing of stomach and intestine was performed with tdTomato + cells isolated from male Bap $x 1^{+/ C r e} ;$ Rosa $26^{+/ t d T o m a t o}$ mice (5 weeks old). Mesenchymal cells were isolated from the glandular hindstomach, which includes both the corpus and the antrum, and used for stomach scRNA-Seq, while mesenchymal cells from the whole intestine were used for intestinal scRNA-Seq. Mesenchymal cell isolation from the stomach and intestinal tissues was modified from the previously described method $^{55}$. Mice were dissected in ice-cold Wash Buffer; HBSS (Gibco) with 2\% (vol/vol) of fetal calf serum (Gibco) and $10 \mathrm{mM}$ HEPES (15630-080, Gibco). Intestines were cut into small pieces and moved to pre-warmed Predigestion Buffer: $30 \mathrm{ml}$ of HBSS with $10 \%$ (vol/vol) inactivated FBS (080-105, Wisent Bioproducts), $10 \mathrm{mM}$ HEPES and $5 \mathrm{mM}$ EDTA (15575-038, Invitrogen). This solution was then incubated for $20 \mathrm{~min}$ at $37^{\circ} \mathrm{C}$ under rotation $(220 \mathrm{rpm})$ in a thermal incubator in a $50 \mathrm{ml}$ tube, followed by filtering through a $100 \mu \mathrm{m}$ cell strainer. The flow-through contains stomach or intestinal contents decanted off epithelial cells. These gut pieces were incubated for $20 \mathrm{~min}$ at $37^{\circ} \mathrm{C}$ under slow rotation, followed by filtering through a $70 \mu \mathrm{m}$ cell strainer. After wash off the remaining EDTA with Wash Buffer, the tissues were collected into $50 \mathrm{ml}$ tubes containing $20 \mathrm{ml}$ of Digestion Buffer; 1\% (vol/vol) penicillin-streptomycin antibiotics (P4333, Sigma), $10 \%$ inactivated FBS, $15 \mathrm{mM}$ HEPES, $100 \mathrm{U} / \mathrm{ml}$ of DNaseI (LS002139, Worthington), $25 \mathrm{U} / \mathrm{ml}$ of collagenase IV (LS004186, Worthington), $0.3 \mathrm{~g} / 100 \mathrm{ml}$ of Dispase (04942078001, Roche). The tissues were digested by incubation at $37^{\circ} \mathrm{C}$ for $30 \mathrm{~min}$ under slow rotation. After vortexing the cell solution intensely for $20 \mathrm{~s}$ every 10 minutes, the tissues were passed through a $40 \mu \mathrm{m}$ cell strainer. After collecting them into $50 \mathrm{ml}$ tubes with $20 \mathrm{ml}$ fresh Digestion Buffer, the previous incubation process was repeated. After combining the supernatants from digestion steps and centrifugation for $5 \mathrm{~min}$ at $400 \mathrm{~g}$ at $20^{\circ} \mathrm{C}$, the pellets were resuspended in Wash Buffer for FACS. CytoxBlue (S34857, Invitrogen) staining was used to distinguish dead cells. tdTomato-positive cells were sorted using MoFlo Astrios (Beckman Coulter).

Drop sequencing. Drop-Seq was performed as described, using a Dolomite Bio (Royston, UK) single-cell RNA-Seq system ${ }^{25}$. Three mice were used to obtain 500,000 of cells for 4000 stamps of drop-seq. The finally recovered cells numbered 4946 for the stomach and 3459 for the intestine each. The viability and number of sorted cells were checked by performing the trypan blue (15250061, Invitrogen) exclusion test prior to Drop-Seq. These cells were then diluted at a concentration of $14 \mathrm{~K}$ cells $/ \mathrm{ml}$ into PBS $+0.01 \%$ BSA. Barcoded Beads SeqB (ChemGenes Corp., Wilmington, MA, USA) were diluted at a concentration of 200 particles/ $\mu \mathrm{L}$ into $200 \mathrm{mM}$ Tris pH 7.5, 6\% Ficoll PM-400, 0.2\% Sarkosyl, 20 mM EDTA, 50 mM DTT.

Quality control of single-cell RNA-Seq data. The quality control metrics for the single-cell RNA-Seq data were obtained using the RNA-SeQC tool (v1.1.7) ${ }^{56}$. This program accepts aligned files as input and delivers a series of plots and statistics for each sample. The quality of the raw reads is defined by a probability distribution across each base call.

Data clustering. DGE expression matrices were processed and analyzed using the $\mathrm{R}$ package Seurat. Only genes expressed in at least 3 cells and cells with minimum 
a
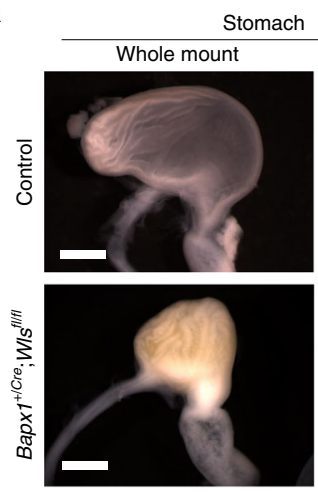
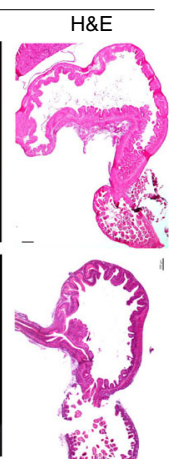

b

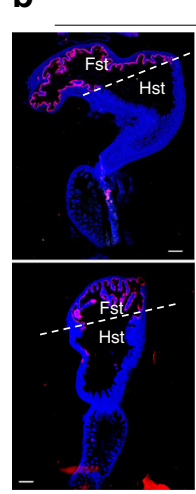

p63/DAPI

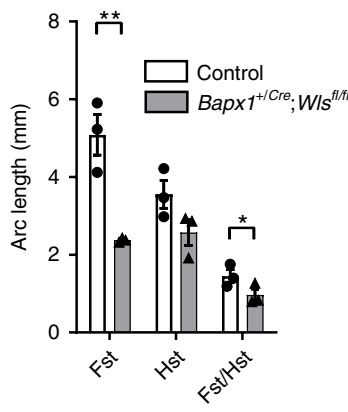

C

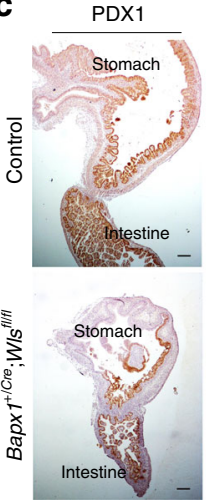

d

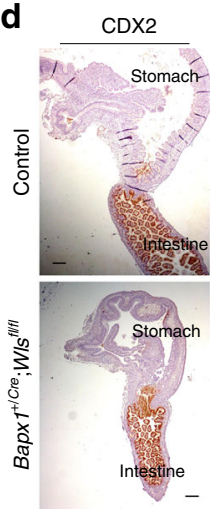

e

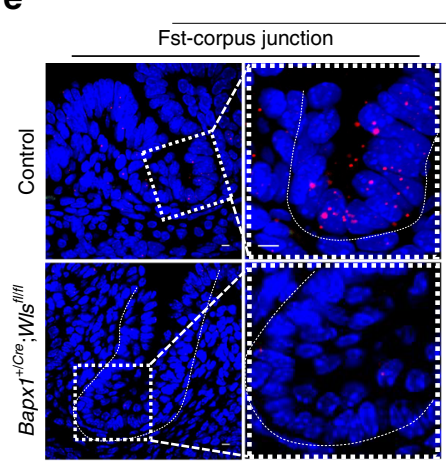

Lgr5/DAPI

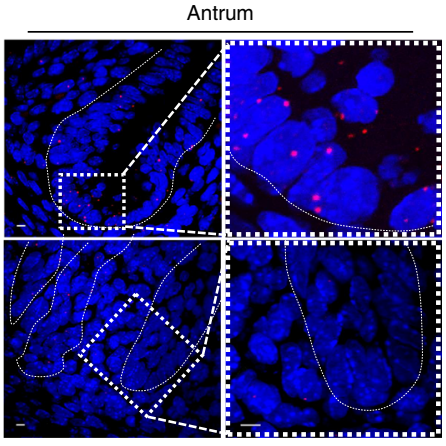

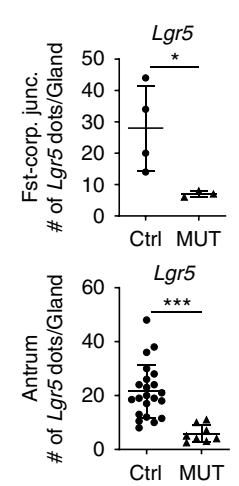

$\mathbf{f}$

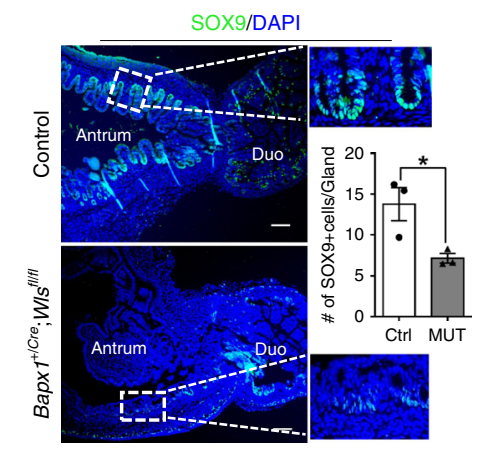

g
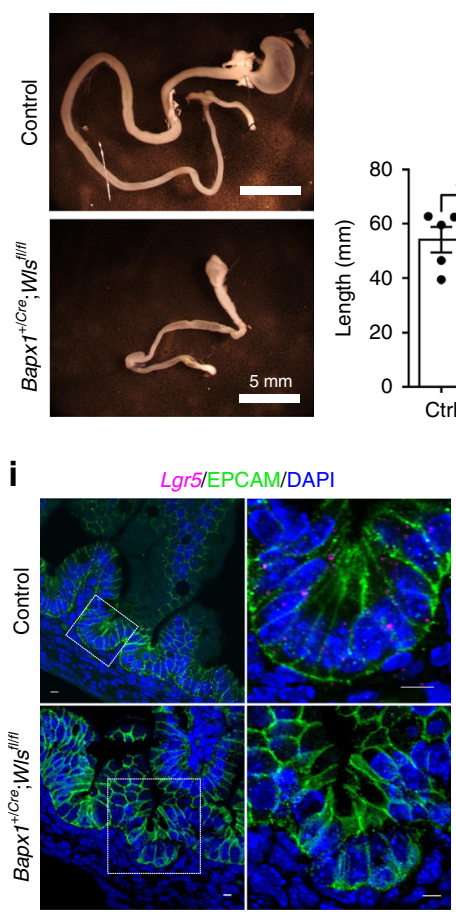

h

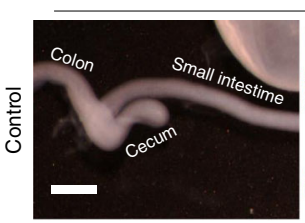

Small intestine
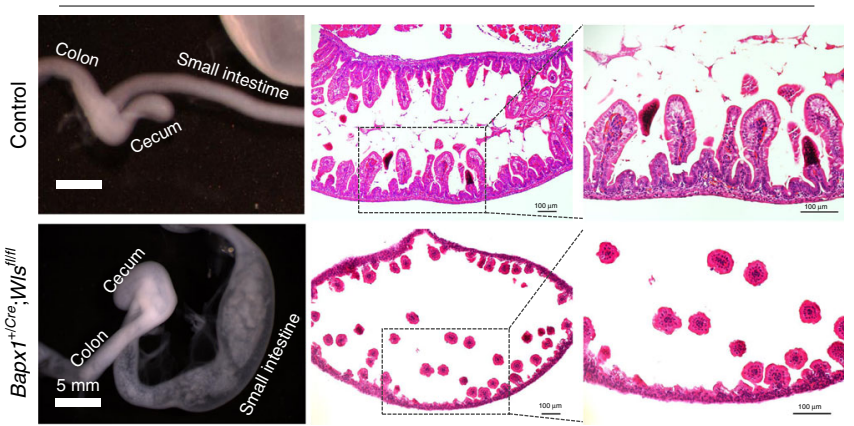
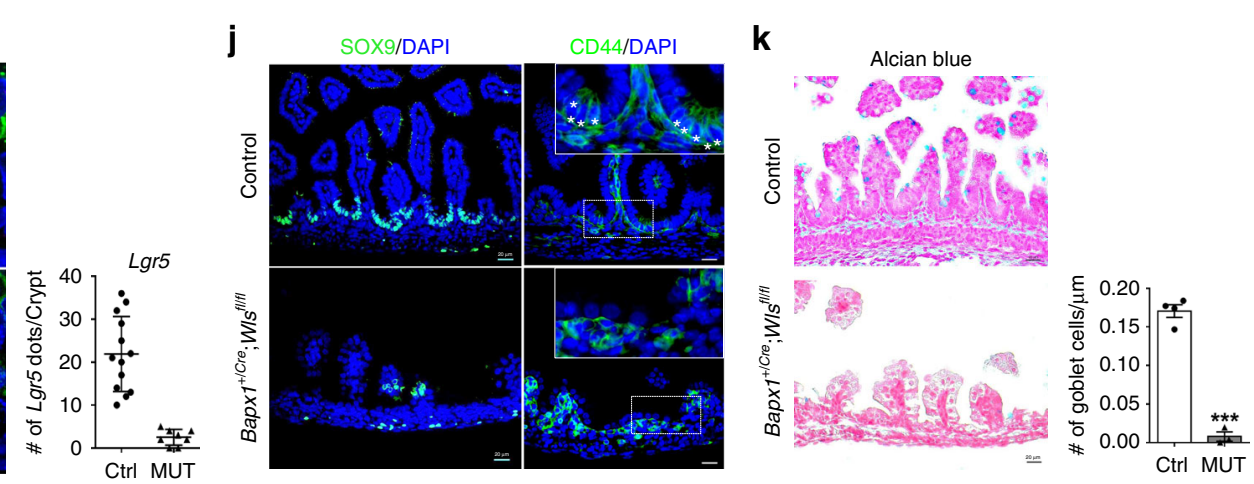

gene numbers of 100 for the stomach and 150 for the intestine were kept for downstream clustering. We chose approximately 2000 highly variable genes to project the scaled cpm (counts per million) normalized data into low dimensional subspace and perform component analysis. Significant PCs were selected before the curve of the standard deviations reached the plateau. Two-dimensional t-SNE was used for visualization. Clustering was based on density clustering. A likelihoodratio test was used to find the differentially expressed genes for identify classes ${ }^{57}$. To overcome the dropout effect in single cell data, we used MAGIC as previously described $^{26}$. We ran the MAGIC with the same parameters $(k=10, a=15)$ in both stomach and intestinal data sets. For better visualization, we used the recovered data set to access the expression levels of Wnt and other gut stromal marker genes, which were projected into the feature plot in R package Seurat. For the coexpression feature plot, we used the mean value as the cutoff to determine whether the gene is expressed in one cell or not.

Cell types comparison between the stomach and intestine. We used an average expression level of the top 30 marker genes as the feature for each cluster in both 
Fig. 7 Stromal Wnt secretion is essential for gastrointestinal development. a Whole mount and H\&E stained images of control and Bapx $1^{+/ C r e} ; \mathrm{Wl} \mid \mathrm{f} / \mathrm{fl}$ stomachs. $\mathbf{b}$ The arc length of forestomach (Fst) and hindstomach (Hst) measured by imageJ shows a significant reduction of the Fst region in Bapx $7^{+/ C r e}$; WIs ${ }^{f l / f l}$ mice compared to the controls. The p63 IF staining (red) demarcates Fst and Hst regions. Scale bars indicate $100 \mu \mathrm{m}$. c, d IHC of PDX1 (c) and CDX2 (d) show a properly maintained stomach-intestinal boundary. Scale bars indicate $100 \mu \mathrm{m}$. e smFISH of Lgr5 in Fst-Corpus junction and Antrum. Red dots indicate $\operatorname{Lgr} 5$ transcripts. Number of $\operatorname{Lgr} 5$ transcripts were significantly reduced in Bapx $1^{+/ C r e}$; Wls fl/fl mice compared to the controls. Scale bars indicate $5 \mu \mathrm{m}$. ( $n=3,1-4$ images per mouse were counted for Lgr5 expression) (f) IF of SOX9 shows a significantly reduced number of SOX 9 expressing cells in the antrum of Bapx $1^{+/ C r e} ; W \mid s^{f l / f l}$ mice, compared to the controls. $n=3 .{ }^{*} p<0.05$. Scales indicate $200 \mu \mathrm{m}$. $\mathbf{g}, \mathbf{h}$ Whole mount $(\mathbf{g})$ and H\&E stained images (h) of control and Bapx $1^{+/ C r e} ; W \mid s^{f l / f l}$ intestines. The intestinal length was dramatically decreased in Bapx $1^{+/ C r e} ; W \mid s^{f l / f l}$ mice compared to the controls. Scale bars indicate $100 \mu \mathrm{m} . n=4$. i smFISH of Lgr5 (magenta dots) with EPCAM IF staining (an epithelial marker; green) in the intestines. The number of Lgr5+ magenta dots (transcripts) were significantly reduced in Bapx ${ }^{+/ / C r e} ; \mathrm{WIs} f / / f l$ mice compared to controls. Scale bars indicate $5 \mu \mathrm{m}$. $(n=3,4-5$ images per mice were counted for Lgr5 expression) (j) IF of SOX9 (left-green) and CD44 (right-green) show their severely impaired expression in Bapx $1^{+/ C r e}$; $W / s^{f l / f l}$

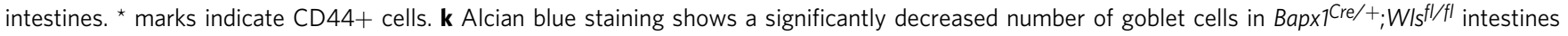
compared to the controls, $n=3,{ }^{\star \star \star \star} P<0.0001$. For all figures, all embryos were analyzed at E17.5. Values are mean \pm SEM. ${ }^{\star} P<0.05$, ${ }^{\star \star} P<0.01,{ }^{\star \star \star} P<$ 0.001. $P$ values were determined using nonparametric unpaired Student's $t$ test. Each $n$ means biologically independent animals and experiments.

the stomach and intestine. Then we calculated the Spearman correlations to compare cell types. A correlation of 0.7 was used to select matching cell types in the stomach and intestine. For further validation, supervised MetaNeighbor ${ }^{58}$ with highly variable genes from the function"get_variable_genes" was used to calculate the AUROC across each cell type from both stomach and intestine.

GSEA analysis. We used average expression of each cluster for analysis with GSEA (version 3.0.0) ${ }^{59,60}$. All the default options were used for GSEA.

Histology, immunohistochemistry, and immunofluorescence. Mouse intestines were dissected in cold $1 \times$ PBS and fixed overnight in $4 \%$ paraformaldehyde (PFA) at $4{ }^{\circ} \mathrm{C}$. The tissues were processed, embedded in paraffin blocks, and sectioned at $4 \mu \mathrm{m}$. For histology, the tissue sections were deparaffinized in xylene, rehydrated through alcohol gradients, and stained with hematoxylin and eosin (Sigma), Alkaline phosphatase substrate (ThermoFisher Scientific), Alcian blue (Sigma) and/ or nuclear fast red (Sigma). The cover slips were then mounted using CytoSeal Mounting Medium (Electron Microscopy Science). For immunohistochemistry (IHC)-Paraffin sections, antigens were retrieved in $10 \mathrm{mM}$ sodium citrate buffer (pH6.0). These sections were permeabilized with $0.3 \%$ Triton X-100 (Sigma), blocked with $5 \%$ fetal donkey serum (Gibco) and Avidin/Biotin Blocking kit (Vector), and incubated overnight with anti-Lysozyme C (Santa Cruz, 1:200) or anti-Pdx1 (Developmental Studies Hybridoma Bank, 1:300). After washing followed by incubation with biotin-conjugated anti-goat, or mouse antibody (Vector Laboratories, 1:300), the staining was developed by using the DAB Substrate Kit (SK-4100, Vector Laboratories). For immunofluorescence (IF)-Paraffin sections, anti-Cdx2 (MU392A-UC, Biogenex, 1:300), anti-BrdU (BDB347580, Fisher scientific, 1:200), anti-CD44 (550538, BD pharmigen, 1:100), anti-Sox9 (AB5535MI, EMD Millipore, 1:200), anti-p63 (619001, BioLegend, 1:300), anti-H,K-ATPase (D032-3H, MBL, 1:400), anti-pHH3 (05-806, Millipore, 1:200) or anti-PCNA (18-0110, Invitrogen, 1:200) was directly incubated overnight after the permeabilization and blocking steps described above. After washing, sections were incubated with Alexa Fluor 594-, 488-conjugated anti-rabbit, rat or mouse IgG (Invitrogen). CD44 immunostaining was enhanced by using the Tyramide Signal Application kit (Perkin-Elmer). For IF-Frozen sections, the tissues embedded in optimal cutting temperature (OCT) compound (Sakura) were sectioned into $10 \mu \mathrm{m}$ and washed with $1 \times$ PBS. After permeabilization and blocking with $0.3 \%$ Triton X-100, $5 \%$ fetal donkey serum (Gibco) for $1 \mathrm{~h}$, the tissue sections were incubated overnight with anti-aSMA (ab124964, Abcam, 1:2000), anti-Desmin (Ab32362, Abcam, 1:1000), anti-PDGFR $\beta$ (Ab32570, Abcam, 1:100), anti-PDGFRa (SC-338, Santa Cruz, 1:200), anti-Nestin (Ab6142, Abcam, 1:200), anti-s100ß (ab41548, Abcam, 1:200), anti-GFAP (Z0334, Dako, 1:300), anti-Lyvel (11-034, Angiobio, 1:400), anti-CD34 (ab81289, abcam, 1:300), anti-CD31 (550274, BD Bioscience, 1:300). E-Cadherin (610183, BD Bioscience, 1:300) or anti-RFP (600-401-379, Rockland Inc, 1:300) antibodies. The cover slips were then mounted using Fluoroshield Mounting Medium with DAPI (ab104139, Abcam). The slides were reviewed using a Nikon E1000 microscope with NIS-Elements BR 4.12.01 imaging software.

Quantitative reverse-transcription PCR (qRT-PCR). RNA was extracted from FACS sorted cells using RNeasy Plus micro kit (74034, QIAGEN), followed by cDNA synthesis with the SuperScript III First-Strand synthesis kit (11752050, ThermoFisher Scientific), according to the manufacturer's instructions. Transcript levels were determined using SYBR Green PCR master mix and the ViiA7 system (4368577, Applied Biosystems). qRT-PCR assays were conducted in triplicates and normalized to the Actin transcript level. The comparative CT method was used to analyze the data. The primer list is shown in Supplementary Table 1.

Small-molecule fluorescence in situ hybridization (smFISH). Mice were perfused with $2 \%$ PFA and fixed overnight in $4 \%$ PFA at $4{ }^{\circ} \mathrm{C}$. The tissues were washed with $1 \times$ PBS three times for $30 \mathrm{~min}$, moved to $30 \%$ sucrose, and incubated overnight. The tissues embedded in OCT were sectioned, and we performed the manufacturer's protocol for single-molecule FISH that is described in the RNAscope Multiplex Fluorescent Detection Kit v2 (323110, ACDBio). The RNAscope Probe (ACDBio)-Mm-Wnt2b (405031-C2), Wnt4 (401101-C2), Wnt2 (313601), Foxl1 (407401), Rspo3 (402011-C3), Rspo1 (479591-C2), Lgr5 (312171-C3), or Shh (314361-C2) was used for single-molecule FISH. The images were acquired with a Nikon A1R Confocal microscope.

Luciferase reporter assay. Stomach and intestinal mesenchymal primary cells were cultured as previously reported ${ }^{61}$ and co-electroporated with $W n t 2 b$ or Wnt9a luciferase, and pRL-TK control plasmids (E2240, Promega), with or without the Gli2 expression plasmid. Electroporation was performed using NEON (Invitrogen; Voltage $1400 \mathrm{~V}$, pulse width $30 \mathrm{~ms}$ and pulse number 1). These cells were incubated in antibiotics-free culture medium (DMEM supplemented with $10 \%$ fetal bovine serum, $1 \%$ non-essential amino acid and $1 \% \mathrm{~L}$-Glutamine) $24 \mathrm{~h}$ prior to electroporation, which was replaced with fresh culture medium containing antibiotics $24 \mathrm{~h}$ after electroporation. They were then harvested 36 hours postelectroporation and lysed to determine luciferase activity, using the Dual Luciferase Reporter Assay System (E0910, Promega) and the Lumat LB 9507 luminometer (ThermoFisher Scientific).

Quantification and statistical analysis. All results were performed in duplicates or triplicates, and expressed as mean \pm S.E.M.; $P$ values were determined using nonparametric unpaired Student's $t$ test (Mann-Whitney $U$ test). $P<0.05$ was considered as statistically significant.

Random forest based similarity learning for scRNA-seq data. We used random forest based similarity learning (RAFSIL) to learn cell-cell similarities of different cell types in each tissue and verify the clustering result from the SNN algorithm. RAFSIL implements a two-step procedure, where feature construction geared towards scRNA-seq data is followed by similarity learning. We chose the top 20 marker genes of each cluster as input features. We modified RAFSIL implementation by pooling data from every 20 cells randomly selected within each cell type and tissue, then applying RAFSIL to the average gene expression profile of the pooled data. We also replaced the randomForest() in RAFSIL with randomForest () in paralleRandomForest. These two simple modifications effectively increase data quality, while reducing the computational burden. paralleRandomFores

Cell type specific expression of disease-associated genes. Using normalized expression values, we obtained a $z$-score for each cell, then computed the mean $z$ score for each cluster. Genes that display any cluster specific-enrichment $(z>1.65)$ were kept for further analysis. We acquired disease-associated SNPs and their neighboring genes from the NHGRI-EBI catalog (https://www.ebi.ac.uk/gwas/48 and plotted the $z$-transformed expression of genes for each disease term individually. Genes were clustered using hclust function (R package: stats) with default parameters, while cell clusters were plotted in the order described above.

Reporting summary. Further information on research design is available in the Nature Research Reporting Summary linked to this article.

\section{Data availability}

The single-cell RNA-seq, bulk RNA-seq and ChIP-seq data set generated for the current study is available in the GEO (Gene Expression Omnibus) database repository: Accession number for single-cell RNA-seq: GSE116514. Accession numbers for RNA-seq data: GSE114450 (stomach), GSE103683 (intestine). Accession numbers for ChIP-seq data: GSE114449 (stomach), GSE103690 (intestine). The source data underlying Figs. 2d, 3c-g, 
4b, c, 4g-j, 5b-f, 6h, i, 7b, and 7e-k, and Supplementary Figs. 4a, b, 20, 22c-e, 23a, b, 24a, b, 25b, 28a, b, and 31 are provided as a Source Data file.

Received: 17 November 2018; Accepted: 14 December 2019; Published online: 17 January 2020

\section{References}

1. Mizuno, T. \& Yasugi, S. Susceptibility of epithelia to directive influences of mesenchymes during organogenesis: uncoupling of morphogenesis and cytodifferentiation. Cell Differ. Dev. 31, 151-159 (1990).

2. Le Guen, L., Marchal, S., Faure, S. \& de Santa Barbara, P. Mesenchymalepithelial interactions during digestive tract development and epithelial stem cell regeneration. Cell Mol. Life Sci. 72, 3883-3896 (2015).

3. Koike, T. \& Yasugi, S. In vitro analysis of mesenchymal influences on the differentiation of stomach epithelial cells of the chicken embryo. Differentiation 65, 13-25 (1999).

4. Kim, B. M., Buchner, G., Miletich, I., Sharpe, P. T. \& Shivdasani, R. A. The stomach mesenchymal transcription factor Barx1 specifies gastric epithelial identity through inhibition of transient Wnt signaling. Dev. Cell 8, 611-622 (2005).

5. Mills, J. C. \& Shivdasani, R. A. Gastric epithelial stem cells. Gastroenterology 140, 412-424 (2011).

6. Barker, N., van de Wetering, M. \& Clevers, H. The intestinal stem cell. Genes Dev. 22, 1856-1864 (2008).

7. Gregorieff, A. \& Clevers, H. Wnt signaling in the intestinal epithelium: from endoderm to cancer. Genes Dev. 19, 877-890 (2005).

8. Kim, T. H. \& Shivdasani, R. A. Stomach development, stem cells and disease. Development 143, 554-565 (2016).

9. McCracken, K. W. et al. Wnt/beta-catenin promotes gastric fundus specification in mice and humans. Nature 541, 182-187 (2017).

10. Bartfeld, S. \& Koo, B. K. Adult gastric stem cells and their niches. Wiley Interdiscip. Rev. Dev. Biol. 6, e261 (2017).

11. Sato, T. et al. Paneth cells constitute the niche for Lgr5 stem cells in intestinal crypts. Nature 469, 415-418 (2011).

12. Farin, H. F., Van, Es,J. H. \& Clevers, H. Redundant sources of Wnt regulate intestinal stem cells and promote formation of Paneth cells. Gastroenterology 143, 1518-1529 e1517 (2012).

13. Kim, T. H., Escudero, S. \& Shivdasani, R. A. Intact function of Lgr5 receptorexpressing intestinal stem cells in the absence of Paneth cells. Proc. Natl Acad. Sci. USA 109, 3932-3937 (2012).

14. Durand, A. et al. Functional intestinal stem cells after Paneth cell ablation induced by the loss of transcription factor Math1 (Atoh1). Proc. Natl Acad. Sci. USA 109, 8965-8970 (2012).

15. San Roman, A. K., Jayewickreme, C. D., Murtaugh, L. C. \& Shivdasani, R. A. Wnt secretion from epithelial cells and subepithelial myofibroblasts is not required in the mouse intestinal stem cell niche in vivo. Stem Cell Rep. 2, 127-134 (2014).

16. Kabiri, Z. et al. Stroma provides an intestinal stem cell niche in the absence of epithelial Wnts. Development 141, 2206-2215 (2014).

17. Valenta, T. et al. Wnt ligands secreted by subepithelial mesenchymal cells are essential for the survival of intestinal stem cells and gut homeostasis. Cell Rep. 15, 911-918 (2016).

18. Aoki, R. et al. Foxl1-expressing mesenchymal cells constitute the intestinal stem cell niche. Cell. Mol. Gastroenterol. Hepatol. 2, 175-188 (2016).

19. Greicius, G. et al. PDGFRalpha $(+)$ pericryptal stromal cells are the critical source of Wnts and RSPO3 for murine intestinal stem cells in vivo. Proc. Nat Acad. Sci. USA 115, E3173-E3181 (2018).

20. Stzepourginski, I. et al. CD34+ mesenchymal cells are a major component of the intestinal stem cells niche at homeostasis and after injury. Proc. Natl Acad. Sci. USA 114, E506-E513 (2017).

21. Shoshkes-Carmel, M. et al. Subepithelial telocytes are an important source of Wnts that supports intestinal crypts. Nature 557, 242-246 (2018).

22. Degirmenci, B., Valenta, T., Dimitrieva, S., Hausmann, G. \& Basler, K. GLI1expressing mesenchymal cells form the essential Wnt-secreting niche for colon stem cells. Nature 558, 449-453 (2018)

23. Sigal, M. et al. Stromal R-spondin orchestrates gastric epithelial stem cells and gland homeostasis. Nature 548, 451-455 (2017).

24. Kim, T. H., Kim, B. M., Mao, J., Rowan, S. \& Shivdasani, R. A. Endodermal Hedgehog signals modulate Notch pathway activity in the developing digestive tract mesenchyme. Development 138, 3225-3233 (2011).

25. Macosko, E. Z. et al. Highly parallel genome-wide expression profiling of individual cells using nanoliter droplets. Cell 161, 1202-1214 (2015).

26. van Dijk, D. et al. Recovering gene interactions from single-cell data using data diffusion. Cell 174, 716-729 e727 (2018).
27. Armulik, A., Genove, G. \& Betsholtz, C. Pericytes: developmental, physiological, and pathological perspectives, problems, and promises. Dev. Cell 21, 193-215 (2011).

28. Banziger, C. et al. Wntless, a conserved membrane protein dedicated to the secretion of Wnt proteins from signaling cells. Cell 125, 509-522 (2006).

29. Barker, N. et al. Lgr5 $(+\mathrm{ve})$ stem cells drive self-renewal in the stomach and build long-lived gastric units in vitro. Cell Stem Cell 6, 25-36 (2010).

30. Leushacke, M. et al. Lgr5-expressing chief cells drive epithelial regeneration and cancer in the oxyntic stomach. Nat. Cell Biol. 19, 774-786 (2017).

31. Pinto, D., Gregorieff, A., Begthel, H. \& Clevers, H. Canonical Wnt signals are essential for homeostasis of the intestinal epithelium. Genes Dev. 17, 1709-1713 (2003).

32. Ireland, $H$. et al. Inducible Cre-mediated control of gene expression in the murine gastrointestinal tract: effect of loss of beta-catenin. Gastroenterology 126, 1236-1246 (2004).

33. Tian, H. et al. Opposing activities of Notch and Wnt signaling regulate intestinal stem cells and gut homeostasis. Cell Rep. 11, 33-42 (2015).

34. Tanno, B. et al. Nanog-driven cell-reprogramming and self-renewal maintenance in Ptch1 (+/-) granule cell precursors after radiation injury. Sci. Rep. 7, 14238 (2017).

35. Kolterud, A. et al. Paracrine Hedgehog signaling in stomach and intestine: new roles for hedgehog in gastrointestinal patterning. Gastroenterology 137, 618-628 (2009).

36. Huang, H. et al. Specific requirement of Gli transcription factors in Hedgehogmediated intestinal development. J. Biol. Chem. 288, 17589-17596 (2013).

37. Mao, J., Kim, B. M., Rajurkar, M., Shivdasani, R. A. \& McMahon, A. P Hedgehog signaling controls mesenchymal growth in the developing mammalian digestive tract. Development 137, 1721-1729 (2010).

38. Schuijers, J., van der Flier, L. G., van Es, J. \& Clevers, H. Robust cre-mediated recombination in small intestinal stem cells utilizing the olfm 4 locus. Stem Cell Rep. 3, 234-241 (2014).

39. Sashikawa Kimura, M., Mutoh, H. \& Sugano, K. SOX9 is expressed in normal stomach, intestinal metaplasia, and gastric carcinoma in humans. $J$. Gastroenterol. 46, 1292-1299 (2011).

40. Khurana, S. S. et al. The hyaluronic acid receptor CD44 coordinates normal and metaplastic gastric epithelial progenitor cell proliferation. J. Biol. Chem. 288, 16085-16097 (2013).

41. Briscoe, J. \& Therond, P. P. The mechanisms of Hedgehog signalling and its roles in development and disease. Nat. Rev. Mol. Cell Biol. 14, 416-429 (2013).

42. Coquenlorge, S. et al. GLI2 modulated by SUFU and SPOP induces intestinal stem cell niche signals in development and tumorigenesis. Cell Rep. 27, 3006-3018 (2019).

43. Whyte, W. A. et al. Master transcription factors and mediator establish superenhancers at key cell identity genes. Cell 153, 307-319 (2013).

44. Merico, D., Isserlin, R., Stueker, O., Emili, A. \& Bader, G. D. Enrichment map: a network-based method for gene-set enrichment visualization and interpretation. PLoS ONE 5, e13984 (2010).

45. Kawazoe, Y. et al. Region-specific gastrointestinal Hox code during murine embryonal gut development. Dev. Growth Differ. 44, 77-84 (2002).

46. Takamoto, N. et al. COUP-TFII is essential for radial and anteroposterior patterning of the stomach. Development 132, 2179-2189 (2005).

47. Jayewickreme, C. D. \& Shivdasani, R. A. Control of stomach smooth muscle development and intestinal rotation by transcription factor BARX1. Dev. Biol. 405, 21-32 (2015)

48. MacArthur, J. et al. The new NHGRI-EBI Catalog of published genome-wide association studies (GWAS Catalog). Nucleic Acids Res. 45, D896-D901 (2017).

49. Gao, N., White, P. \& Kaestner, K. H. Establishment of intestinal identity and epithelial-mesenchymal signaling by Cdx2. Dev. Cell 16, 588-599 (2009).

50. Barker, N. et al. Identification of stem cells in small intestine and colon by marker gene Lgr5. Nature 449, 1003-1007 (2007).

51. Zhao, C. et al. Stromal Gli2 activity coordinates a niche signaling program for mammary epithelial stem cells. Science 356, 1003-1007 (2017).

52. Yauch, R. L. et al. A paracrine requirement for hedgehog signalling in cancer. Nature 455, 406-410 (2008).

53. Chee, Y. C. et al. Intrinsic xenobiotic resistance of the intestinal stem cell niche. Dev. Cell 46, 681-695 e685 (2018).

54. Faussone-Pellegrini, M. S. \& Gherghiceanu, M. Telocyte's contacts. Semin. Cell Dev. Biol. 55, 3-8 (2016).

55. Weigmann, B. et al. Isolation and subsequent analysis of murine lamina propria mononuclear cells from colonic tissue. Nat. Protoc. 2, 2307-2311 (2007).

56. DeLuca, D. S. et al. RNA-SeQC: RNA-seq metrics for quality control and process optimization. Bioinformatics 28, 1530-1532 (2012).

57. McDavid, A. et al. Data exploration, quality control and testing in single-cell qPCR-based gene expression experiments. Bioinformatics 29, 461-467 (2013).

58. Crow, M., Paul, A., Ballouz, S., Huang, Z. J. \& Gillis, J. Characterizing the replicability of cell types defined by single cell RNA-sequencing data using MetaNeighbor. Nat. Commun. 9, 884 (2018). 
59. Mootha, V. K. et al. PGC-1alpha-responsive genes involved in oxidative phosphorylation are coordinately downregulated in human diabetes. Nat. Genet 34, 267-273 (2003).

60. Yamashita, S. The post-embedding method for immunoelectron microscopy of mammalian tissues: a standardized procedure based on heat-induced antigen retrieval. Methods Mol. Biol. 657, 237-248 (2010).

61. Koliaraki, V. \& Kollias, G. Isolation of intestinal mesenchymal cells from adult mice. Bio-protocol 6, e1940 (2016).

\section{Acknowledgements}

This work has been supported by SickKids Foundation start-up, the University of Toronto's Medicine by Design Initiative/the Canada First Research Excellence Fund, the Canadian Institutes of Health Research (CIHR; PJT 155923) and Natural Science Research Council of Canada (NSERC) Discovery \#RGPIN-2016-06093 grants (T.H.K.); the CIHR and the University of Toronto's Medicine by Design postdoctoral fellowships (J.E.K.); the CIHR (PJT 162083) (H.K.S.); the SickKids Restracomp graduate student fellowships (A.R.B. and N.A.H.); the NSERC Discovery and CIHR grants (C.C.H.). We thank Dr. Warren Zimmer for Bap $x 1^{C r e}$ mice and Dr. Troy Katela at the Princess Margaret Genomics Centre for performing Drop-Seq. We thank Edward Espionza for helpful discussion.

\section{Author contributions}

J.E.K. conceived, designed, and performed the experiments, analyzed the data and drafted the paper; L.F. performed the bioinformatics analyses for single cell transcriptomes and analyzed data; W.C.Y. performed GLI2 ChIP-Seq with stomach tissue and bioinformatic analyses; S.C.G. performed GLI2 ChIP-Seq with stomach tissue; A.R.B. analyzed mice with pericyte-like stromal cell-specific Hh gain and loss of functions; X.Z. performed some GLI2 ChIP-Seq related experiments; S.W.S. and J.H.L. helped with CyTOF analysis; N.H. analyzed cell type specific expression of diseaseassociated genes; W.R. helped with some mouse studies; K.H.K. helped with $\mathrm{Ng} 2$ reporter mouse analysis; H.K.S. supervised CyTOF analysis; C.C.H. provided Hh signaling mouse lines and supervised bioinformatic analyses; G.G. supervised single cell analyses and analyzed data; T.H.K. conceived and supervised the study, analyzed data, and wrote the paper.

\section{Competing interests}

The authors declare no competing interests.

\section{Additional information}

Supplementary information is available for this paper at https://doi.org/10.1038/s41467019-14058-5.

Correspondence and requests for materials should be addressed to T.-H.K.

Peer review information Nature Communications thanks the anonymous reviewer(s) for their contribution to the peer review of this work.

Reprints and permission information is available at http://www.nature.com/reprints

Publisher's note Springer Nature remains neutral with regard to jurisdictional claims in published maps and institutional affiliations.

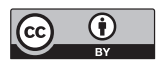

Open Access This article is licensed under a Creative Commons Attribution 4.0 International License, which permits use, sharing, adaptation, distribution and reproduction in any medium or format, as long as you give appropriate credit to the original author(s) and the source, provide a link to the Creative Commons license, and indicate if changes were made. The images or other third party material in this article are included in the article's Creative Commons license, unless indicated otherwise in a credit line to the material. If material is not included in the article's Creative Commons license and your intended use is not permitted by statutory regulation or exceeds the permitted use, you will need to obtain permission directly from the copyright holder. To view a copy of this license, visit http://creativecommons.org/licenses/by/4.0/.

(C) The Author(s) 2020 